\title{
Bounding the Influence of Domain Parameterization and Knot Spacing on Numerical Stability in Isogeometric Analysis
}

\author{
Elisabeth Pilgerstorfer ${ }^{1}$, Bert Jüttler \\ Institute of Applied Geometry, Johannes Kepler University, Linz, Austria
}

\begin{abstract}
Isogeometric Analysis (IGA) was introduced by Hughes et al. in 2005 [1] as a new method to bridge the gap between the geometry description and numerical analysis. Similar to the finite element approach, the IGA concept to solve a partial differential equation leads to a (linear) system of equations. The condition number of the coefficient matrix is a crucial factor for the stability of the system. It depends strongly on the domain parameterization, which provides the isogeometric discretization. In this paper we derive a bound for the condition number of the stiffness matrix of the Poisson equation. In particular, we investigate the influence of the domain parameterization and the knot spacing on the stability of the numerical system. The factors appearing in our bound reflect the stability properties of a given domain parameterization.
\end{abstract}

Keywords: numerical stability, Isogeometric Analysis, domain parameterization, knot spacing, condition number, quality measure

\section{Introduction}

The concept of Isogeometric Analysis (IGA) was first proposed by Hughes et al. [1] in 2005 to provide a seamless integration of Computer-Aided Design (CAD) and Finite Element Analysis (FEA). In IGA, the same basis functions are used for the geometry description and for the numerical analysis. One major advantage of IGA over the classical finite element method (FEM) is the improved representation of the computational domain by using nonuniform rational B-splines (NURBS) or other classes of basis functions. Furthermore, due

\footnotetext{
${ }^{1}$ Corresponding author.

E-mail address: elisabeth.pilgerstorfer@jku.at
} 
to the increased smoothness of the basis functions, the numerical solution inherits a high continuity.

Since the first publication of Hughes and his co-workers on IGA, an increasing number of researchers worldwide are working in that field, applying the new methodology to a wide variety of simulation problems. Within the last 8 years, 184 papers with the word "isogeometric" in the title have been published alone in the journal Computer Methods in Applied Mechanics and Engineering. The number of publications per year is increasing, which shows that IGA is a very active field of research.

Besides practical issues, also the theoretical foundations of IGA have been analyzed thoroughly. Here we mention a few representative results: Fundamental results on approximation properties, error estimates and numerical stability in IGA are described in [2-4]. Among other issues, these publications derive error estimates for approximation by NURBS functions with respect to degree, smoothness and stepsize (knot spacing). Another important issue is the derivation and analysis of efficient quadrature rules for IGA, see [5].

Especially when dealing with large problems, the computational effort for solving the numerical system becomes an important issue. It is then preferable to use iterative solvers instead of direct solvers to reduce the computational costs. When using an iterative solver, the condition number is a crucial factor, since it highly influences the rate of convergence. Furthermore, small changes in the right-hand side of a linear system could cause big changes in the solution, if the system matrix is not well conditioned.

In the classical FEM literature one can find considerations about the stiffness matrix and bounds for the condition number, being of order $\mathcal{O}\left(h^{-2}\right)$ for uniform discretizations with grid size $h$, see, e.g., [6-8]. This also generalizes to locally refined meshes under mild restrictions on the refinement, see, e.g., [9]. There are also recent publications giving finer bounds for $p$-FEM, see, e.g., [10]. Moreover, it has been analyzed how the quality of the underlying mesh influences the numerical properties of the stiffness matrix, see, e.g., [11] and the references cited therein.

Many results from classical FEM can be carried over to the isogeometric approach. However, when we want to compute bounds for the condition number of the stiffness matrix, some differences occur due to the presence of the geometry mapping and the larger support of the basis functions.

The condition number in IGA depends on the underlying parameterization of the computational domain. Hence, this number can be used to measure the quality of a param- 
eterization. Our goal is to analyze the influence of the parameterization of the domain of interest on the stability of the numerical system. More precisely, our aim is to derive an upper bound for the condition number, which reflects the quality of the domain parameterization.

Construction of domain parameterizations that are suitable for IGA have been presented in several publications. In [12] we provided a tool to construct B-spline or NURBS swept volumes via a variational framework. This class of volumes is suitable to parameterize functional free-form shapes such as blades for turbines and propellers. Moreover, we discussed the influence of the chosen parameterization on the accuracy of the solution. However, we had no simple measure for the quality of a parameterization.

Cohen et al. [13] introduce the framework of analysis-aware modeling, where model properties and parameters should be selected to facilitate isogeometric analysis. Martin et al. [14] provide a method to construct volumetric B-spline parameterizations from input genus-0 triangle meshes using harmonic functions.

In $[15-17]$ the authors show that the parameterization of the computational domain has an impact on the simulation result and the efficiency of the computations. An optimal parameterization of the computational domain is generated by a shape optimization method. Additionally, an easy-to-check algorithm to ensure that the constructed parameterization has no self-intersections is proposed.

The articles $[18,19]$ analyze the influence of singularities in the parameterization of the physical domain. The authors present regularity results and modification schemes for the test function space in the case of reduced regularity. In [20] a method for shape optimization using the Winslow functional is introduced. In [21] and [22] the authors use this technique to optimize the domain of interest for special applications such as vibrating membranes or conducting scatterers.

In [23-26] the authors discuss various methods for shape optimization of different domains. Lipton et al. [27] discovered the effect of severe distortion of the control and physical mesh. In all cases, using higher order basis functions leads to increased robustness under mesh distortion.

In many of these earlier studies, a parameterization was considered to be "good" in the sense that it is analysis-suitable, whenever the physical mesh looks "nice". The ratio of maximum to minimum physical element size should not exceed prescribed limits and the physical elements should not be too much distorted. However, this was a rather heuristic 
way to define a "good" parameterization. As far as we know, there are no publications considering the influence of the parameterization on the stability of the numerical system.

Recently, Gahalaut and Tomar [28] derived estimates for the condition number of the stiffness and mass matrix for IGA for $h$ - and $p$-refinement. Their estimates depend on the polynomial degree of basis functions $p$ and the mesh size $h$ and some constant. The dependence on the geometry mapping $G$, however, is hidden in the constant.

In contrast to this approach, in our paper we derive bounds for the condition number of the stiffness matrix which make explicit the influence of the domain parameterization. The investigated bounds depend on the knot vector and on a term arising from the geometry mapping. Special attention will be paid to the latter term, since this provides a quality measure for the parameterization.

The remainder of this paper is organized as follows: In Section 2 we recall the principles of IGA and introduce our notation. Section 3 describes the outline and the basic steps of our approach to find a bound for the condition number of the stiffness matrix. In the following three sections we will derive bounds for the one-, two- and three-dimensional case. We will present several examples and compare different parameterizations in Section 7. Finally we will conclude the paper in Section 8.

\section{Preliminaries}

We recall the principles of IGA and formulate the model problem. Furthermore, we recall some elementary results about quadratic forms and matrix inequalities.

\subsection{IGA on a single patch}

Consider the unit cube $\Omega_{0}=[0,1]^{D}$ in $\mathbb{R}^{D}$, where $D \in\{1,2,3\}$ is the dimension. The cube $\Omega_{0}$ is called the parameter domain. We denote by $\left\{\beta_{i}^{d}\right\}_{i=1, \ldots, n_{d}}$ the univariate B-splines of degree $p_{d}$ for the $d$-th parameter direction, which are defined by a knot vector

$$
\left[0, \ldots, 0, \ldots, k_{d}^{\ell_{d}}, k_{d}^{\ell_{d}+1}, \ldots, 1, \ldots, 1\right]
$$

where $d=1, \ldots, D$. We will use open knot vectors, i.e., with $\left(p_{d}+1\right)$-fold boundary knots. The symbol $n_{d}$ denotes the total number of B-splines in the $d$-th parameter direction. The length of a knot span $\left[k_{d}^{\ell_{d}}, k_{d}^{\ell_{d}+1}\right]$ is

$$
h_{d}^{\ell_{d}}=k_{d}^{\ell_{d}+1}-k_{d}^{\ell_{d}} .
$$


Throughout this paper we will use the following convention for indices: The lower index refers to the space coordinate, while the upper index identifies the knot span, which will also be called an element. Note that we will use multi-indices for the latter. In particular, in the case $D=1$ we will omit the lower index by simply writing $h^{\ell}$ for the length of the knot $\operatorname{span}\left[k^{\ell}, k^{\ell+1}\right]$.

We introduce the multi-index $\mathbf{i}=\left(i_{1}, \ldots, i_{D}\right)$ and the index set

$$
\mathbb{I}=\left\{\mathbf{i} \in \mathbb{N}^{D}: 0 \leq i_{1} \leq n_{1}, \ldots, 0 \leq i_{D} \leq n_{D}\right\}
$$

The (multivariate) rational (or NURBS) basis functions $\psi_{\mathbf{i}}$ are defined by

$$
\psi_{\mathbf{i}}(\boldsymbol{\xi})=\psi_{\mathbf{i}}\left(\xi_{1}, \ldots, \xi_{D}\right)=\frac{w_{\mathbf{i}} \cdot \beta_{i_{1}}^{1}\left(\xi_{1}\right) \cdot \ldots \cdot \beta_{i_{D}}^{D}\left(\xi_{D}\right)}{\sum_{\mathbf{i}^{\prime} \in \mathbb{I}} w_{\mathbf{i}^{\prime}} \cdot \beta_{i_{1}^{\prime}}^{1}\left(\xi_{1}\right) \cdot \ldots \cdot \beta_{i_{D}^{\prime}}^{D}\left(\xi_{D}\right)}
$$

with certain positive weights $w_{\mathbf{i}}>0$. The basis functions $\psi_{\mathbf{i}}$ will be referred to as geometry basis functions. These basis functions form a convex partition of unity.

Summing up, the rational basis functions are defined by specifying the dimension $D$ of the parameter domain, the $D$ knot vectors and degrees, and a positive weight for each function. We refer to [29-32] for more detailed information on properties and algorithms for B-splines and NURBS.

Throughout this paper we assume that the following two assumptions are satisfied:

Assumption 1 The knot vectors in all parameter directions $d=1, \ldots, D$ are locally quasi-uniform, i.e., there exists a constant $K$ such that the lengths of the knot spans satisfy

$$
\frac{h_{d}^{j_{d}}}{h_{d}^{\ell_{d}}} \leq K, \quad j_{d}=\ell_{d}-p_{d}, \ldots, \ell_{d}+p_{d}
$$

for all indices $\ell_{d}$ with $h_{d}^{\ell_{d}} \neq 0$.

Consequently, for a given non-empty knot span, the lengths of all those knot spans which belong to the support of B-splines whose support includes the given knot span, is bounded from above by a certain constant multiple of the length of the given span.

The local quasi-uniformity of the knot vectors is a condition on the refinement algorithm. The knot vectors should be refined in a way that this condition is fulfilled for some constant $K$. The constant $K$ will be used later in the derivation of the estimates for the condition number. 
Assumption 2 The weights are bounded, i.e., there exists a constant $W \geq 1$ such that

$$
\frac{1}{W} \leq w_{\mathbf{i}} \leq W
$$

holds for all indices $\mathbf{i} \in \mathbb{I}$.

The boundedness of the weights is always preserved when refining a given parameterization, due to the convex hull property of B-spline representations.

In addition to the parameter domain, we consider the physical domain $\Omega \subset \mathbb{R}^{D}$ with boundary $\partial \Omega$. We assume that it is connected to the parameter domain via the geometry mapping

$$
\mathbf{G}: \Omega_{0} \rightarrow \Omega, \quad \xi \mapsto \mathbf{x}=\mathbf{G}(\boldsymbol{\xi}),
$$

which maps the parameter domain $\Omega_{0}$ to the physical domain $\Omega$, see Fig. 1 . The geometry mapping is a linear combination of the rational basis functions,

$$
\mathbf{G}=\sum_{\mathbf{i}} \mathbf{d}_{\mathbf{i}} \psi_{\mathbf{i}}
$$

where $\mathbf{d}_{\mathbf{i}} \in \mathbb{R}^{D}$ are the control points. The mapping $\mathbf{G}$ is assumed to be bijective.
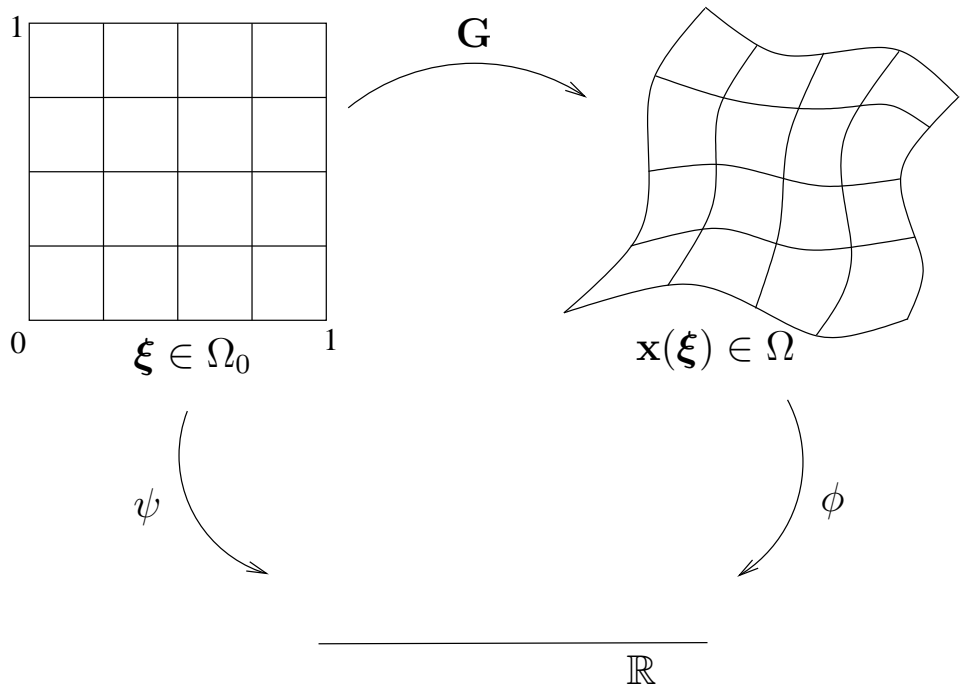

Figure 1: The parameter domain $\Omega_{0}$, the physical domain $\Omega$, the geometry mapping $\mathbf{G}$ connecting the two domains, the geometry basis functions $\psi$ and the isogeometric test functions $\phi$.

IGA is a method for computing an approximate solution of a partial differential equation (PDE) on the domain $\Omega$. The unknown solution $u$ of the PDE is approximated by a function

$$
u_{h}(\mathbf{x})=\sum_{\mathbf{i}} c_{\mathbf{i}} \phi_{\mathbf{i}}(\mathbf{x})
$$


which is a linear combination of isogeometric test functions $\phi_{\mathbf{i}}(\mathbf{x})=\left(\psi_{\mathbf{i}} \circ \mathbf{G}^{-1}\right)(\mathbf{x})$, see Fig. 1, with certain coefficients $c_{\mathbf{i}} \in \mathbb{R}$.

A major advantage of using B-splines or NURBS for the geometry parameterization is the reduction or even elimination of errors resulting from the geometry representation. Furthermore, the increased smoothness of the basis functions with compact support leads to superior approximation properties. Further details of the isogeometric concept are described in $[1,33]$.

\subsection{Quadratic forms and matrix inequalities}

In the following we consider symmetric $m \times m$ matrices with real entries. Consider two matrices $\mathbf{A}$ and $\mathbf{B}$. We write

$$
\mathbf{A} \leq \mathbf{B} \text { if } \forall \mathbf{x} \in \mathbb{R}^{m}: \mathbf{x}^{T} \mathbf{A} \mathbf{x} \leq \mathbf{x}^{T} \mathbf{B} \mathbf{x}
$$

and

$$
\mathbf{A} \geq \mathbf{B} \text { if } \forall \mathbf{x} \in \mathbb{R}^{m}: \mathbf{x}^{T} \mathbf{A} \mathbf{x} \geq \mathbf{x}^{T} \mathbf{B} \mathbf{x} .
$$

Given a matrix $\mathbf{A}$, the Rayleigh quotient $R_{\mathbf{A}}(\mathbf{x})$ is defined as

$$
R_{\mathbf{A}}(\mathbf{x})=\frac{\mathbf{x}^{T} \mathbf{A} \mathbf{x}}{\mathbf{x}^{T} \mathbf{x}}
$$

with $\mathbf{x} \in \mathbb{R}^{m}, \mathbf{x} \neq \overrightarrow{0}$. It satisfies

$$
\lambda_{\min }(\mathbf{A}) \leq R_{\mathbf{A}}(\mathbf{x}) \leq \lambda_{\max }(\mathbf{A}),
$$

where $\lambda_{\min }(\mathbf{A})$ and $\lambda_{\max }(\mathbf{A})$ denote the minimum and the maximum eigenvalue of the matrix A. Consider 4 matrices $\mathbf{A}, \mathbf{A}^{\prime}, \mathbf{B}, \mathbf{B}^{\prime}$ and the identity matrix $\mathbf{I}$. We recall the following simple results:

(i) $\lambda_{\min }(\mathbf{A}) \mathbf{I} \leq \mathbf{A} \leq \lambda_{\max }(\mathbf{A}) \mathbf{I}$.

(ii) $\mathbf{A} \leq \mathbf{B} \quad \Rightarrow \quad \lambda_{\min }(\mathbf{A}) \leq \lambda_{\min }(\mathbf{B})$ and $\lambda_{\max }(\mathbf{A}) \leq \lambda_{\max }(\mathbf{B})$.

(iii) $\left(\mathbf{A} \leq \mathbf{A}^{\prime}\right) \wedge\left(\mathbf{B} \leq \mathbf{B}^{\prime}\right) \Rightarrow \mathbf{A}+\mathbf{B} \leq \mathbf{A}^{\prime}+\mathbf{B}^{\prime}$

(iv) $\lambda_{\max }(\mathbf{A}+\mathbf{B}) \leq \lambda_{\max }(\mathbf{A})+\lambda_{\max }(\mathbf{B})$

Definition 3 A set $\mathcal{A}$ of $m \times m$ matrices $\mathbf{A}$ is said to be bounded, if there exists a constant $C$ such that all elements of all matrices $\mathbf{A}=\left(a_{i, j}\right)_{i, j} \in \mathcal{A}$ satisfy $\left|a_{i, j}\right| \leq C$. Thus, the set $\mathcal{A}$ corresponds to a bounded subset of $\mathbb{R}^{m^{2}}$. 
For later reference we formulate an auxiliary result:

Lemma 4 Consider a bounded set $\mathcal{A}$ of symmetric $m \times m$ matrices. There exist real constants $\underline{\lambda}$ and $\bar{\lambda}$, depending on the chosen set $\mathcal{A}$, such that every matrix $\mathbf{A} \in \mathcal{A}$ satisfies

$$
\underline{\lambda} \mathbf{I} \leq \mathbf{A} \leq \bar{\lambda} \mathbf{I}
$$

where $\mathbf{I}$ is the $m \times m$ identity matrix and the inequalities are considered in the sense of Eq. (2). If the set $\mathcal{A}$ is a bounded and closed set of symmetric and positive definite matrices, then constants $\underline{\lambda}$ and $\bar{\lambda}$ with $0<\underline{\lambda}<\bar{\lambda}$ exist.

Proof. Any matrix $\mathrm{A} \in \mathcal{A}$ can be bounded by

$$
\lambda_{\min }(\mathbf{A}) \mathbf{I} \leq \mathbf{A} \leq \lambda_{\max }(\mathbf{A}) \mathbf{I}
$$

Since the set $\mathcal{A}$ is bounded, we can obtain global bounds which have to satisfy

$$
\underline{\lambda} \leq \inf _{\mathbf{A} \in \mathcal{A}} \lambda_{\min }(\mathbf{A}) \text { and } \bar{\lambda} \geq \sup _{\mathbf{A} \in \mathcal{A}} \lambda_{\max }(\mathbf{A}) .
$$

If the set $\mathcal{A}$ is a bounded and closed set of symmetric and positive definite $m \times m$ matrices, all eigenvalues of the matrices $\mathbf{A}$ in the set are strictly positive, hence the lower bound $\underline{\lambda}$ and the upper bound $\bar{\lambda}$ are strictly positive.

We discuss a simple example of a bounded and closed set $\mathcal{A}$ of symmetric and positive definite matrices: We consider quadratic univariate B-splines defined on a sequence of knots $\left[\ldots, k_{0},-1,1, k_{1}, \ldots\right]$, where $k_{0} \leq-1$ and $k_{1} \geq 1$. On the knot span [-1,1], exactly three basis functions do not vanish. For this element we consider the element mass matrix $\mathbf{M}^{\ell}$ for the identical geometry mapping, which possesses the entries

$$
m_{i, j}^{\ell}=\int_{-1}^{1} \psi_{i} \psi_{j} \mathrm{~d} \xi
$$

see also Eq. (15) in Section 4.1. The element mass matrix $\mathbf{M}^{\ell}$ is a symmetric and positive definite $3 \times 3$ matrix, where each entry depends on the coefficients $k_{0}$ and $k_{1}$. Exemplarily we present the expressions for some of the entries:

$$
\begin{aligned}
& m_{1,1}^{\ell}=\frac{8}{5\left(k_{0}-1\right)^{2}} \\
& m_{1,2}^{\ell}=-\frac{4\left(2+4 k_{0}+k_{1}+5 k_{0} k_{1}\right)}{15\left(k_{0}-1\right)^{2}\left(k_{1}+1\right)} \\
& m_{1,3}^{\ell}=-\frac{4}{15\left(k_{0}-1\right)\left(k_{1}+1\right)}
\end{aligned}
$$


If the knots $k_{0}$ and $k_{1}$ vary in a bounded interval, then the collection of matrices $\mathbf{M}^{\ell}$ satisfy the assumptions of the second part of Lemma 4. Since the element mass matrices are symmetric and positive definite, we can find positive values $\underline{\lambda}$ and $\bar{\lambda}$, such that $\underline{\lambda} \mathbf{I} \leq$ $\mathbf{M}^{\ell} \leq \bar{\lambda} \mathbf{I}$ holds for all matrices $\mathbf{M}^{\ell} \in \mathcal{A}$. For instance, if we restrict the knots $k_{i}$ to $k_{0} \in[-5,-1], k_{1} \in[1,5]$ we obtain $\underline{\lambda}=0.00391081$ and $\bar{\lambda}=1.26276$.

This observation admits the following geometric interpretation: For a fixed pair of values $\left(k_{0}, k_{1}\right)$, the equation $\mathbf{x}^{T} \mathbf{M}^{\ell} \mathbf{x}=1$ describes an ellipsoid. The equation $\mathbf{x}^{T}(\underline{\lambda} \mathbf{I}) \mathbf{x}=1$ describes the smallest sphere, that is circumscribed about all ellipsoids in the set $\mathcal{A}$. The equation $\mathbf{x}^{T}(\bar{\lambda} \mathbf{I}) \mathbf{x}=1$ describes the largest sphere, that is inscribed in all ellipsoids in the set. Fig. 2 shows some of the ellipsoids and the smallest circumscribed and the largest inscribed sphere for the previously defined ranges of $k_{0}$ and $k_{1}$.
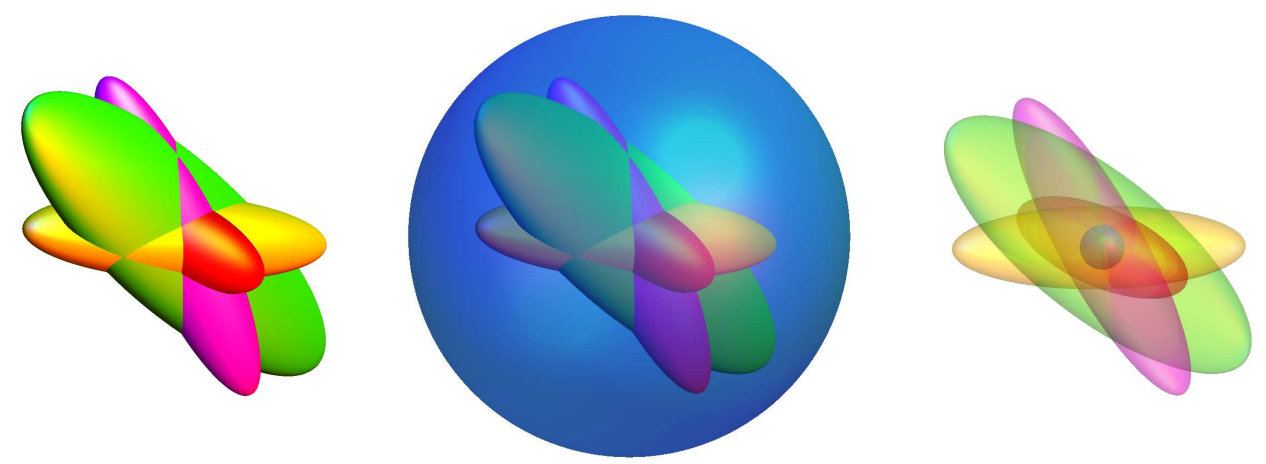

Figure 2: Geometric interpretation of the bounded set of matrices. The symmetric and positive definite $3 \times 3$ matrices $\mathbf{M}^{\ell}$ correspond to ellipsoids, the matrices $\underline{\lambda} \mathbf{I}$ and $\bar{\lambda} \mathbf{I}$ correspond to the minimum subscribed and the maximum inscribed sphere, respectively.

For future reference we recall

Theorem 5 (Cauchy's interlace theorem [34]). Let $\mathbf{A}$ be a symmetric $n \times n$ matrix and $\mathbf{B}$ be a symmetric $m \times m$ sub-matrix constructed from $\mathbf{A}$ by deleting columns and rows with $m \leq n$. Let the eigenvalues of $\mathbf{A}$ and $\mathbf{B}$ be $\alpha_{1} \leq \alpha_{2} \leq \ldots \leq \alpha_{n}$ and $\beta_{1} \leq \beta_{2} \leq \ldots \leq \beta_{m}$, respectively. These eigenvalues satisfy

$$
\alpha_{k} \leq \beta_{k} \leq \alpha_{k+n-m}, \quad k=1, \ldots, m
$$

This Theorem implies

$$
\lambda_{\min }(\mathbf{B}) \geq \lambda_{\min }(\mathbf{A}) \text { and } \lambda_{\max }(\mathbf{B}) \leq \lambda_{\max }(\mathbf{A}) .
$$




\subsection{Model problem, weak formulation, stiffness matrix}

We consider the Poisson equation for the unknown function $u$ with homogeneous Dirichlet boundary conditions over the physical domain $\Omega \subset \mathbb{R}^{D}, D \in\{1,2,3\}$,

$$
\left\{\begin{aligned}
-\Delta u(\mathbf{x}) & =f(\mathbf{x}) & & \text { on } \Omega \\
u(\mathbf{x}) & =0 & & \text { on } \partial \Omega
\end{aligned}\right.
$$

with $\mathbf{x}=\left(x_{1}, \ldots, x_{D}\right), f \in L^{2}(\Omega)$. Applying Galerkin projection to the associated weak formulation leads to the problem to find the approximate solution

$$
u_{h}(\mathbf{x})=\sum_{\mathbf{i}} c_{\mathbf{i}} \phi_{\mathbf{i}}(\mathbf{x})
$$

which satisfies

$$
\forall v_{h} \in V_{0 h}: \underbrace{\int_{\Omega} \nabla u_{h}^{T}(\mathbf{x}) \nabla v_{h}(\mathbf{x}) \mathrm{d} \mathbf{x}}_{=a\left(u_{h}, v_{h}\right)}=\underbrace{\int_{\Omega} f(\mathbf{x}) v_{h}(\mathbf{x}) \mathrm{d} \mathbf{x}}_{=\left(f, v_{h}\right)},
$$

where the test functions form the space

$$
V_{0 h}=\left\{v_{h} \in \operatorname{span}\left\{\phi_{\mathbf{i}}: \mathbf{i} \in \mathbb{I}_{0}\right\}\right\} \text { with } \mathbb{I}_{0}=\left\{\mathbf{i} \in \mathbb{I}: \phi_{\mathbf{i}}=0 \text { on } \partial \Omega\right\} .
$$

By choosing a basis $\left\{\phi_{\mathbf{i}}: \mathbf{i} \in \mathbb{I}_{0}\right\}$ of isogeometric test functions for $V_{0 h}$ we can then derive a linear system of equations

$$
\mathrm{Sc}=\mathbf{f}
$$

for the unknowns $\mathbf{c}=\left(c_{\mathbf{i}}\right)_{\mathbf{i} \in \mathbb{I}_{0}}$, where $\mathbf{S}=\left(a\left(\phi_{\mathbf{i}}, \phi_{\mathbf{j}}\right)\right)_{\mathbf{i}, \mathbf{j} \in \mathbb{I}_{0}}$ is the stiffness matrix and $\mathbf{f}=\left(\left(f, \phi_{\mathbf{i}}\right)\right)_{\mathbf{i} \in \mathbb{I}_{0}}$ is often called the load vector.

The entries of the stiffness matrix $\mathbf{S}$ are given by integrals over products of gradients of basis functions,

$$
s_{\mathbf{i}, \mathbf{j}}=a\left(\phi_{\mathbf{i}}, \phi_{\mathbf{j}}\right)=\int_{\Omega} \nabla \phi_{\mathbf{i}}^{T}(\mathbf{x}) \nabla \phi_{\mathbf{j}}(\mathbf{x}) \mathrm{d} \mathbf{x} .
$$

Using the Jacobian $\mathbf{J}$ of the geometry mapping $\mathbf{G}$ and its determinant

$$
\omega(\boldsymbol{\xi})=|\operatorname{det} \mathbf{J}(\boldsymbol{\xi})|,
$$

the integral over the physical domain $\Omega$ can be transformed into an integral over the parameter domain $\Omega_{0}$, since $\phi=\psi \circ \mathbf{G}^{-1}$. We obtain

$$
s_{\mathbf{i}, \mathbf{j}}=\int_{\Omega_{0}} \nabla \psi_{\mathbf{i}}^{T}(\boldsymbol{\xi}) \mathbf{N}(\boldsymbol{\xi}) \nabla \psi_{\mathbf{j}}(\boldsymbol{\xi}) \mathrm{d} \boldsymbol{\xi} \quad \text { with } \quad \mathbf{N}(\boldsymbol{\xi})=\omega(\boldsymbol{\xi}) \mathbf{J}^{-1}(\boldsymbol{\xi}) \mathbf{J}^{-T}(\boldsymbol{\xi}) .
$$


The matrix $\mathbf{N}$ is a symmetric and positive definite $D \times D$ matrix. In particular, for dimension $D=1$, we obtain

$$
\mathrm{N}(\xi)=\frac{1}{|\dot{G}(\xi)|}
$$

where the dot denotes the derivative with respect to $\xi$. The stiffness matrix $\mathbf{S}$ is symmetric and positive definite. Due to the local support of the basis functions, the stiffness matrix is sparse.

Assumption 6 Throughout this paper we will consider only regular parameterizations of the physical domain, i.e., we assume $\omega(\boldsymbol{\xi}) \neq 0$, see Eq. (5).

\subsection{Element-wise construction the stiffness matrix}

For any realistic problem size, the global stiffness matrix is assembled from element stiffness matrices. Analogous to classical FEM, the element stiffness matrices are given by integrating over an element. In IGA an element is defined as the knot span between two adjacent knot values in the one-dimensional case and as the Cartesian product of knot spans in higher dimensions. Using the multi-index $\ell=\left(\ell_{1}, \ldots, \ell_{D}\right)$, we will denote the elements by $E^{\ell}$,

$$
E^{\ell}=\underset{d=1}{D}\left[k_{d}^{\ell_{d}}, k_{d}^{\ell_{d}+1}\right] .
$$

Note that some of these elements may be empty, as some of the knots may coincide. During the analysis we consider the non-empty elements only.

The element stiffness matrix $\tilde{\mathbf{S}}^{\ell}$ for the element $E^{\ell}$ contains the entries

$$
\tilde{s}_{\mathbf{i}, \mathbf{j}}^{\ell}=\int_{E^{\ell}} \nabla \psi_{\mathbf{i}}^{T}(\boldsymbol{\xi}) \mathbf{N}(\boldsymbol{\xi}) \nabla \psi_{\mathbf{j}}(\boldsymbol{\xi}) \mathrm{d} \boldsymbol{\xi} .
$$

Many entries of the element stiffness matrices vanish due to the local support of the basis functions. Therefore we define reduced element stiffness matrices $\mathbf{S}^{\ell}$, where we store only the relevant entries, i.e., we restrict the index set to the indices of the functions whose support contains the element. We obtain the full element stiffness matrix $\tilde{\mathbf{S}}^{\ell}$ from the reduced ones by multiplication with element contribution matrices $\left(\mathbf{P}^{\ell}\right)^{T}$ and $\mathbf{P}^{\ell}$,

$$
\tilde{\mathbf{S}}^{\ell}=\left(\mathbf{P}^{\ell}\right)^{T} \mathbf{S}^{\ell}\left(\mathbf{P}^{\ell}\right)
$$

The element contribution matrices $\mathbf{P}^{\ell}$ can be easily constructed by considering the diagonal entries of the element stiffness matrices. If the diagonal entry in position $(\mathbf{i}, \mathbf{i})$ of the reduced 
element stiffness matrix $\mathbf{S}^{\ell}$ is in position $(\mathbf{j}, \mathbf{j})$ in the full element stiffness matrix $\tilde{\mathbf{S}}^{\ell}$, we will have the entry 1 in position $(\mathbf{i}, \mathbf{j})$ in the element contribution matrix $\mathbf{P}^{\ell}$. All other entries of $\mathbf{P}^{\ell}$ are 0 .

The global stiffness matrix $\mathbf{S}_{\text {glob }}$ is obtained by summing the full element stiffness matrices,

$$
\mathrm{S}_{\mathrm{glob}}=\sum_{\ell} \tilde{\mathbf{S}}^{\ell}
$$

Finally, the homogeneous Dirichlet boundary conditions are incorporated by deleting the corresponding columns and rows of the global stiffness matrix $\mathbf{S}_{\text {glob }}$. The stiffness matrix with incorporated Dirichlet boundary conditions will be denoted as $\mathbf{S}$.

The reduced element stiffness matrices $\mathbf{S}^{\ell}$ are symmetric and positive semi-definite. The global stiffness matrix $\mathbf{S}$ with incorporated Dirichlet boundary conditions is symmetric and positive definite. Table 1 shows the dimensions of the matrices introduced so far.

\begin{tabular}{c|l|c|c} 
matrix & description & rows & columns \\
\hline $\mathbf{S}_{\text {glob }}$ & global stiffness matrix & $\prod_{d=1}^{D} n_{d}$ & $\prod_{d=1}^{D} n_{d}$ \\
& global stiffness matrix with incorpo- & $\prod_{d=1}^{D}\left(n_{d}-2\right)$ & $\prod_{d=1}^{D}\left(n_{d}-2\right)$ \\
$\mathbf{S}$ & rated Dirichlet boundary conditions & $\prod_{d=1}^{D} n_{d}$ & $\prod_{d=1}^{D} n_{d}$ \\
$\tilde{\mathbf{S}}^{\ell}$ & full element stiffness matrix & $\prod_{d=1}^{D}\left(p_{d}+1\right)$ & $\prod_{d=1}^{D}\left(p_{d}+1\right)$ \\
$\mathbf{S}^{\ell}$ & reduced element stiffness matrix & $\prod_{d=1}^{D}\left(p_{d}+1\right)$ & $\prod_{d=1}^{D} n_{d}$ \\
$\mathbf{P}^{\ell}$ & element contribution matrix & $d$
\end{tabular}

Table 1: Dimensions of the matrices used to construct the global stiffness matrix.

Remark 7 Note that in practice the assembling of the global stiffness matrix is a little different. One starts with computing the reduced element stiffness matrices. With a connectivity matrix of size $n_{d} \times \prod_{d=1}^{D}\left(p_{d}+1\right)$, which holds the global numbering, one assembles the global system matrix. This approach is less memory consuming than our proposed approach by computing full and reduced element matrices and connecting them via element contribution matrices. However, our approach leads to a very simple and hence appealing relation between the involved matrices which may be preferable for the theoretical analysis.

Remark 8 In the sequel we will need only the reduced element stiffness matrices. For the sake of brevity we will omit the word "reduced" and call these matrices simply element 
stiffness matrices.

\subsection{Element-wise bounds of geometry-related terms}

In the one-dimensional case $(D=1)$, we abbreviate the maximum and the minimum of the function $\omega=|\dot{G}|$ on the element $E^{\ell}$ by $\omega_{\max }^{\ell}$ and $\omega_{\min }^{\ell}$ respectively, i.e.,

$$
\omega_{\max }^{\ell}=\max _{\xi \in E^{\ell}}|\dot{G}(\xi)| \text { and } \quad \omega_{\min }^{\ell}=\min _{\xi \in E^{\ell}}|\dot{G}(\xi)|
$$

In higher dimensions $(D>1)$, we need the element-wise maximum of the absolute value of each entry of the matrix $\mathbf{N}$,

$$
N_{i, j, \max }^{\ell}=\max _{\boldsymbol{\xi} \in E^{\ell}}\left|N_{i, j}(\boldsymbol{\xi})\right|
$$

and we assemble these in another matrix

$$
\mathbf{N}_{\max }^{\ell}=\left(N_{i, j, \max }^{\ell}\right)_{i, j=1, \ldots, D}
$$

In addition, we generalize the one-dimensional notation (7) to

$$
\omega_{\max }^{\ell}=\max _{\boldsymbol{\xi} \in E^{\ell}} \omega(\boldsymbol{\xi}) \text { and } \omega_{\min }^{\ell}=\min _{\boldsymbol{\xi} \in E^{\ell}} \omega(\boldsymbol{\xi}) .
$$

Assumption 9 We assume that for every element there exists a positive constant $\delta^{\ell}$ such that the determinant of the Jacobian satisfies

$$
\forall \boldsymbol{\xi} \in E^{\ell}: \omega(\boldsymbol{\xi}) \geq \delta^{\ell} \omega_{\max }^{\ell}
$$

The constants $\delta^{\ell}$ are given by

$$
\delta^{\ell}=\frac{\omega_{\min }^{\ell}}{\omega_{\max }^{\ell}} .
$$

In the case $D=1$, this inequality can be rewritten as

$$
\forall \xi \in E^{\ell}:|\dot{G}(\xi)| \geq \delta^{\ell} \omega_{\max }^{\ell}
$$

with the constant $\delta^{\ell}=\frac{\omega_{\min }^{\ell}}{\omega_{\max }^{\ell}}$.

Note that uniform refinement of a given isogeometric discretization implies that all $\delta^{\ell}$ tend to 1. 


\section{Analysis of the condition number}

This section derives a bound of the condition number in terms of matrices which are defined on the elements of the isogeometric discretization. First we provide an outline of the approach. We consider the maximum and the minimum eigenvalues separately, where the analysis of the latter one requires us to introduce the concept of mass matrices. Finally we formulate the bound for the condition number.

\subsection{Outline of our approach}

Since the global stiffness matrix with incorporated Dirichlet boundary conditions is symmetric and positive definite, the condition number $\kappa(\mathbf{S})$ is given by

$$
\kappa(\mathbf{S})=\frac{\lambda_{\max }(\mathbf{S})}{\lambda_{\min }(\mathbf{S})}
$$

In order to derive an upper bound for the condition number $\kappa$, we need to bound the maximum eigenvalue from above and the minimum eigenvalue from below. The estimates are derived in several steps, which follow the classical approach to derive these bounds for the finite element method. Fig. 3 summarizes the estimates used to construct a bound for the condition number.

First, the maximum eigenvalue of the global stiffness matrix is bounded by the maximum of the maximum eigenvalues of the element stiffness matrices. For each element stiffness matrix, its maximum eigenvalue is bounded by a term depending on the geometry mapping and another term depending on the knot vector.

On the other hand, the minimum eigenvalue of the global stiffness matrix is bounded by the minimum eigenvalue of the global mass matrix, using the Friedrichs inequality. Next, the procedure is the same as for the stiffness matrix. The minimum eigenvalue of the global mass matrix is bounded by the minimum of the minimum eigenvalues of the element mass matrices. For each element mass matrix, its minimum eigenvalue is bounded by a term depending on the geometry mapping and another term which depends on the knot vector. The bound for the minimum eigenvalue is bounded away from 0 . The details of the estimates are presented in the following sections.

\subsection{Bounding the maximum eigenvalue}

We start by considering the maximum eigenvalue of the stiffness matrix. 


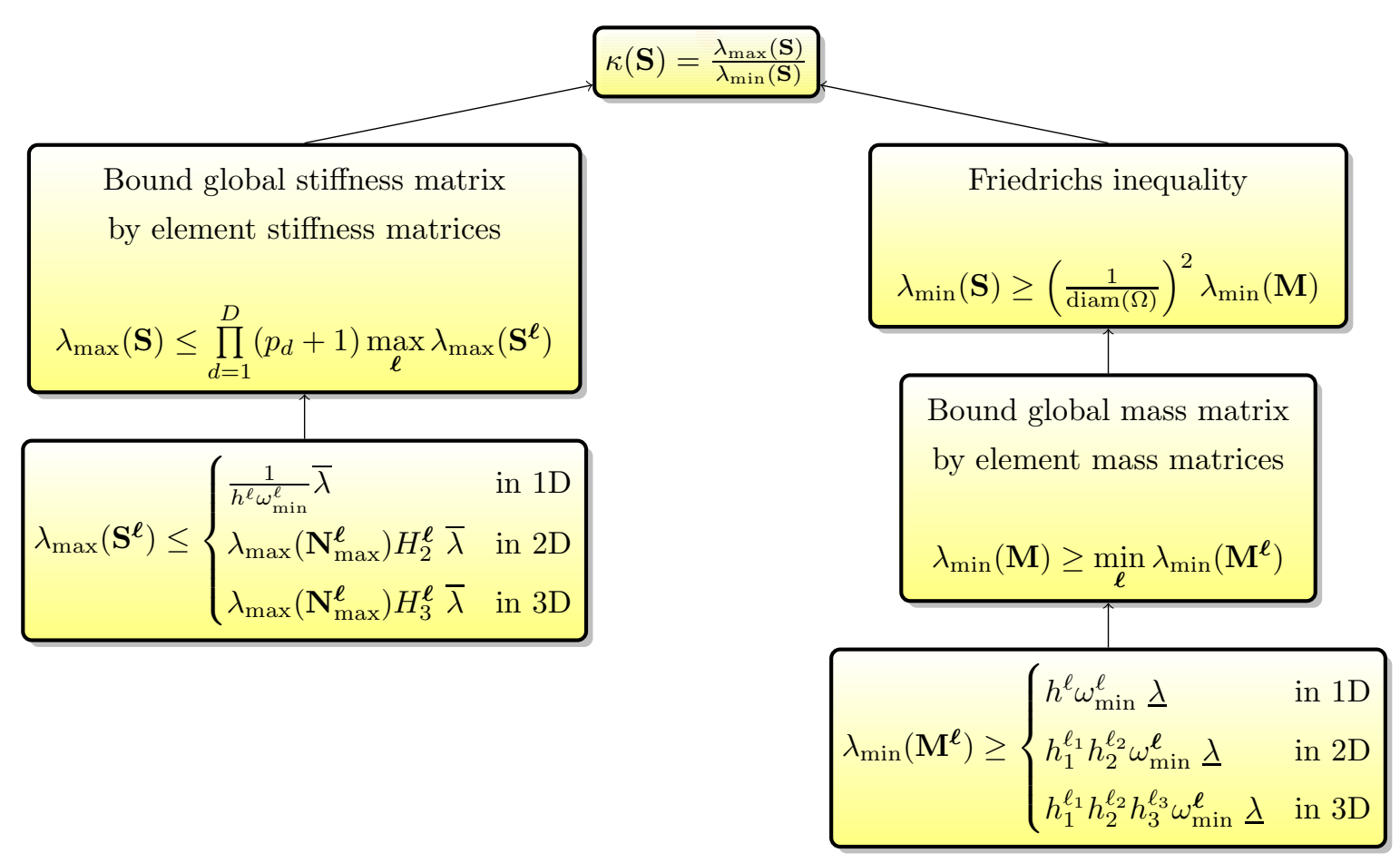

Figure 3: Illustration of the estimates used to construct a bound for the condition number of the stiffness matrix. The expressions for the variables $H_{2}^{\ell}$ and $H_{3}^{\ell}$ are defined in Eq. (23) for dimension $D=2$ and in Eq. (30) for dimension $D=3$.

Lemma 10 The maximum eigenvalues of the element stiffness matrices provide an upper bound of the maximum eigenvalue of the global stiffness matrix,

$$
\lambda_{\max }(\mathbf{S}) \leq\left(\prod_{d=1}^{D}\left(p_{d}+1\right)\right) \max _{\ell} \lambda_{\max }\left(\mathbf{S}^{\ell}\right) .
$$

Proof. Since the element stiffness matrices $\mathbf{S}^{\ell}$ are symmetric and positive semi-definite, we can bound the matrices from above by

$$
\mathbf{S}^{\ell} \leq \lambda_{\max }\left(\mathbf{S}^{\ell}\right) \mathbf{I}
$$

The global stiffness matrix $\mathbf{S}_{\text {glob }}$ without incorporated Dirichlet boundary conditions is assembled from the element stiffness matrices by

$$
\mathbf{S}_{\text {glob }}=\sum_{\ell}\left(\mathbf{P}^{\ell}\right)^{T} \mathbf{S}^{\ell} \mathbf{P}^{\ell}
$$

Inserting the matrix bound for $\mathbf{S}^{\ell}$, we obtain

$$
\mathbf{S}_{\mathrm{glob}} \leq \sum_{\ell}\left(\mathbf{P}^{\ell}\right)^{T} \lambda_{\max }\left(\mathbf{S}^{\ell}\right) \mathbf{I} \mathbf{P}^{\ell}
$$


where the identity matrix $\mathbf{I}$ in this equation has the same dimensions as the element stiffness matrix. The maximum number of terms of the form $\lambda_{\max }\left(\mathbf{S}^{\ell}\right)$ in the diagonal of this matrix is

$$
\prod_{d=1}^{D}\left(p_{d}+1\right)
$$

where $p_{d}$ is the degree of the B-spline/NURBS basis functions in the $d$-th parameter direction. This implies

$$
\mathbf{S}_{\text {glob }} \leq\left(\prod_{d=1}^{D}\left(p_{d}+1\right)\right) \max _{\ell} \lambda_{\max }\left(\mathbf{S}^{\ell}\right) \mathbf{I}
$$

where the identity matrix $\mathbf{I}$ in this equation has the same dimensions as the global stiffness matrix $\mathbf{S}_{\text {glob. From this it follows }}$

$$
\lambda_{\max }\left(\mathbf{S}_{\text {glob }}\right) \leq \lambda_{\max }\left(\left(\prod_{d=1}^{D}\left(p_{d}+1\right)\right) \max _{\ell} \lambda_{\max }\left(\mathbf{S}^{\ell}\right) \mathbf{I}\right)=\left(\prod_{d=1}^{D}\left(p_{d}+1\right)\right) \max _{\ell} \lambda_{\max }\left(\mathbf{S}^{\ell}\right) .
$$

The global stiffness matrix $\mathbf{S}$ with incorporated Dirichlet boundary conditions is constructed by deleting columns and rows of $\mathbf{S}_{\text {glob }}$. Hence we know by Cauchy's interlace theorem and Eq. (4) that

$$
\lambda_{\max }(\mathbf{S}) \leq \lambda_{\max }\left(\mathbf{S}_{\text {glob }}\right)
$$

which concludes the proof.

\subsection{The Friedrichs inequality and the mass matrix}

The element stiffness matrices are singular. Thus we cannot use the element stiffness matrices to bound the minimum eigenvalue of the global stiffness matrix. Similar to the approach in the classical FEM literature we address this problem by using the Friedrichs inequality:

Lemma 11 (Friedrichs inequality [35]) Consider a bounded subset $\Omega$ of $\mathbb{R}^{D}$ with diameter $\operatorname{diam}(\Omega)$ and an element $u$ of the Sobolev space $W_{0}^{k, q}(\Omega)$. Then the following inequality holds:

$$
\|u\|_{L^{q}(\Omega)} \leq(\operatorname{diam}(\Omega))^{k}\left(\sum_{|\alpha|=k}\left\|D^{\alpha} u\right\|_{L^{q}(\Omega)}^{q}\right)^{\frac{1}{q}} .
$$

In particular, for $q=2$ and $k=1$ we obtain

$$
\|u\|_{L^{2}(\Omega)} \leq \operatorname{diam}(\Omega)\|\nabla u\|_{L^{2}(\Omega)} .
$$


For further investigation we need to define the mass matrix $\mathbf{M}$. The entries $m_{\mathbf{i}, \mathbf{j}}$ of the mass matrix $\mathbf{M}$ are given by integrals over products of basis functions, i.e.,

$$
m_{\mathbf{i}, \mathbf{j}}=\int_{\Omega} \phi_{\mathbf{i}}(\mathbf{x}) \phi_{\mathbf{j}}(\mathbf{x}) \mathrm{d} \mathbf{x} .
$$

Similar to the stiffness matrix, the entries of the mass matrix can be transformed to integrals over the parameter domain $\Omega_{0}$ by

$$
\int_{\Omega} \phi_{\mathbf{i}}(\mathbf{x}) \phi_{\mathbf{j}}(\mathbf{x}) \mathrm{d} \mathbf{x}=\int_{\Omega_{0}} \psi_{\mathbf{i}}(\boldsymbol{\xi}) \psi_{\mathbf{j}}(\boldsymbol{\xi}) \omega(\boldsymbol{\xi}) \mathrm{d} \boldsymbol{\xi} .
$$

All entries of the mass matrix are non-negative, since the basis functions are non-negative everywhere. The mass matrix is symmetric and positive definite. The global mass matrix can be constructed by summing up element mass matrices,

$$
\mathbf{M}_{\text {glob }}=\sum_{\ell} \tilde{\mathbf{M}}^{\ell}
$$

with

$$
\tilde{\mathbf{M}}^{\ell}=\left(\tilde{m}_{\mathbf{i}, \mathbf{j}}^{\ell}\right)_{\mathbf{i}, \mathbf{j}} \text { and } \tilde{m}_{\mathbf{i}, \mathbf{j}}^{\ell}=\int_{E^{\ell}} \psi_{\mathbf{i}}(\boldsymbol{\xi}) \psi_{\mathbf{j}}(\boldsymbol{\xi}) \omega(\boldsymbol{\xi}) \mathrm{d} \boldsymbol{\xi}
$$

By deleting certain columns and rows of $\mathbf{M}_{\text {glob }}$ to incorporate the homogeneous Dirichlet boundary conditions we obtain the matrix $\mathbf{M}$.

Similar to the construction of the stiffness matrix we define reduced element mass matrices $\mathbf{M}^{\ell}$ that just contain the relevant entries,

$$
\tilde{\mathbf{M}}^{\ell}=\left(\mathbf{P}^{\ell}\right)^{T} \mathbf{M}^{\ell} \mathbf{P}^{\ell}
$$

The reduced element mass matrices are symmetric and positive definite. Again we will omit the term "reduced" and call these matrices just element mass matrices.

Corollary 12 The smallest eigenvalue of the stiffness matrix is bounded from below by the smallest eigenvalue of the mass matrix,

$$
\lambda_{\min }(\mathbf{S}) \geq \frac{1}{(\operatorname{diam}(\Omega))^{2}} \lambda_{\min }(\mathbf{M}) .
$$

Proof. Using the Friedrichs inequality, we obtain

$$
\mathbf{c}^{T} \mathbf{S c}=\left\|\nabla u_{h}\right\|^{2} \geq \frac{1}{(\operatorname{diam}(\Omega))^{2}}\left\|u_{h}\right\|^{2}=\frac{1}{(\operatorname{diam}(\Omega))^{2}} \mathbf{c}^{T} \mathbf{M c} .
$$

The minimum eigenvalues of the matrices $\mathbf{S}$ and $\mathbf{M}$ are the minima of the corresponding Rayleigh quotients. This implies (12). 


\subsection{Bounding the minimum eigenvalue}

We consider the contribution of each element to the mass matrix:

Lemma 13 The minimum eigenvalue of the global mass matrix can be bounded by the minimum eigenvalue of the element mass matrices,

$$
\lambda_{\min }(\mathbf{M}) \geq \min _{\ell} \lambda_{\min }\left(\mathbf{M}^{\ell}\right)
$$

where the minimum is taken over all non-empty elements.

Proof. Since the element mass matrices $\mathbf{M}^{\ell}$ are symmetric and positive definite, we can bound the matrices from below by

$$
\mathbf{M}^{\ell} \geq \lambda_{\min }\left(\mathbf{M}^{\ell}\right) \mathbf{I}
$$

which implies

$$
\left(\mathbf{P}^{\ell}\right)^{T} \mathbf{M}^{\ell} \mathbf{P}^{\ell} \geq\left(\mathbf{P}^{\ell}\right)^{T} \lambda_{\min }\left(\mathbf{M}^{\ell}\right) \mathbf{I} \mathbf{P}^{\ell}
$$

By definition, the global mass matrix $\mathbf{M}_{\text {glob }}$ is assembled from the element mass matrices, see Eq. (10) and (11). Using the matrix bound for $\mathbf{M}^{\ell}$ we obtain

$$
\mathbf{M}_{\text {glob }} \geq \sum_{\ell}\left(\mathbf{P}^{\ell}\right)^{T} \lambda_{\min }\left(\mathbf{M}^{\ell}\right) \mathbf{I} \mathbf{P}^{\ell}
$$

The matrix on the right-hand side of this inequality is a diagonal matrix. Since at least one term of the form $\lambda_{\min }\left(\mathbf{M}^{\ell}\right)$ contributes to each diagonal entry, we conclude that

$$
\mathbf{M}_{\mathrm{glob}} \geq \min _{\ell} \lambda_{\min }\left(\mathbf{M}^{\ell}\right) \mathbf{I}
$$

where the identity matrix in this equation has the same dimensions as the global mass matrix $\mathbf{M}_{\text {glob }}$. Furthermore, we obtain

$$
\lambda_{\min }\left(\mathbf{M}_{\mathrm{glob}}\right) \geq \lambda_{\min }\left(\min _{\ell} \lambda_{\min }\left(\mathbf{M}^{\ell}\right) \mathbf{I}\right)=\min _{\ell} \lambda_{\min }\left(\mathbf{M}^{\ell}\right)
$$

Since the global mass matrix $\mathbf{M}$ with incorporated Dirichlet boundary conditions is constructed by deleting columns and rows of $\mathbf{M}_{\text {glob }}$, we can use Cauchy's interlace theorem and Eq. (4) to conclude the proof. 


\subsection{Putting things together}

Now we are ready to formulate the main result of this section.

Theorem 14. The condition number of the stiffness matrix is bounded by

$$
\kappa(\mathbf{S}) \leq(\operatorname{diam}(\Omega))^{2}\left(\prod_{d=1}^{D}\left(p_{d}+1\right)\right) \frac{\max _{\ell} \lambda_{\max }\left(\mathbf{S}^{\ell}\right)}{\min _{\ell} \lambda_{\min }\left(\mathbf{M}^{\ell}\right)}
$$

Proof. This result is proved by combining Corollary 12, Lemma 10 and Lemma 13.

Thus we have to analyze the maximum eigenvalues of the element stiffness matrices and the minimum eigenvalues of the element mass matrices.

For the bound of the condition number we will consider the different cases of dimension $D$ individually. The basic idea is to transform the element stiffness matrices and the element mass matrices to a standard element and to bound the resulting matrices using compactness results.

\section{The case $D=1$}

This section considers the case $D=1$. While this special case enables us to explain the main steps of our approach, it allows for some simplifications. Indeed, the geometry mapping $G$ and its Jacobian are scalar-valued functions, thus $\omega=|\dot{G}|$.

The next section shows how to transform the integrals and the basis functions to the unit interval. The following two sections derive the bound for the eigenvalues of the element stiffness and mass matrices. The results then allow us to formulate a bound for the condition number of the global stiffness matrix in Section 4.4.

\subsection{Transformation to the unit interval}

When we use B-splines/NURBS of polynomial degree $p$ as basis functions, $p+1$ basis functions are defined on each non-empty element $E^{\ell}=\left[k^{\ell}, k^{\ell+1}\right]$. The element stiffness and mass matrices $\mathbf{S}^{\ell}=\left(s_{i, j}^{\ell}\right)_{i, j}$ and $\mathbf{M}^{\ell}=\left(m_{i, j}^{\ell}\right)_{i, j}$ have the entries

$$
s_{i, j}^{\ell}=\int_{k^{\ell}}^{k^{\ell+1}} \frac{\dot{\psi}_{i}(\xi) \dot{\psi}_{j}(\xi)}{\omega(\xi)} \mathrm{d} \xi \quad \text { and } \quad m_{i, j}^{\ell}=\int_{k^{\ell}}^{k^{\ell+1}} \psi_{i}(\xi) \psi_{j}(\xi) \omega(\xi) \mathrm{d} \xi
$$

respectively. The dot denotes the derivative with respect to the variable $\xi$. 
The element stiffness and mass matrices for the element $E^{\ell}$ depend on $2 p+1$ knot spans with lengths $h^{\ell-p}, h^{\ell-p+1}, \ldots, h^{\ell}, \ldots, h^{\ell+p}$. Fig. 4 illustrates the notation of the knots and lengths of knot spans. Note that we could also have multiple knots, e.g., $k^{i}=k^{i+1}$, which lead to a vanishing knot span $h^{i}=0$.

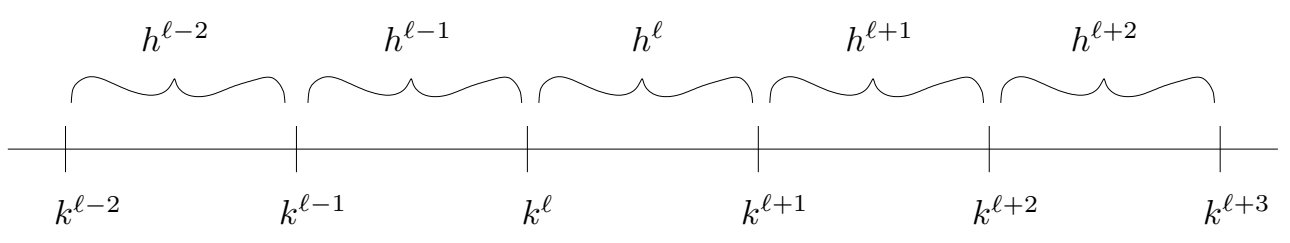

Figure 4: Illustration of knots and lengths of knot spans.

In the NURBS case, the element stiffness and mass matrices depend additionally on $p+1$ weights $w_{i}, w_{i+1}, \ldots, w_{i+p}$ corresponding to the $p+1$ NURBS basis functions $\psi_{i}$, $\psi_{i+1}, \ldots, \psi_{i+p}$ defined on $E^{\ell}$.

Given an element $E^{\ell}$, we will transform the integrals from $E^{\ell}$ to the unit element $[0,1]$. We will denote the transformed functions defined on the unit element by

$$
\hat{\psi}_{i}=\psi_{i} \circ \alpha^{\ell}, \quad \hat{\dot{G}}^{\ell}=\dot{G} \circ \alpha^{\ell}
$$

etc., where $\alpha^{\ell}$ is the affine mapping

$$
\alpha^{\ell}: \mathbb{R} \rightarrow \mathbb{R}: \quad \zeta \mapsto \xi(\zeta)=h^{\ell} \zeta+k^{\ell}
$$

which satisfies $\alpha^{\ell}(0)=k^{\ell}$ and $\alpha^{\ell}(1)=k^{\ell+1}$. Note that $\hat{\psi}_{i}$ depends also on the number $\ell$ of the given element. In order to keep the notation simple, we omitted the index $\ell$ here.

Fig. 5 illustrates the mapping $\alpha^{\ell}$, which maps the unit element to the element $E^{\ell}$. In particular it shows how the mapping $\alpha^{\ell}$ modifies the knots specifying the NURBS basis functions $\psi_{i}$. The knots are scaled and translated such that the knot span $\left[k^{\ell}, k^{\ell+1}\right]$ becomes the unit interval $[0,1]$.

The argument of the transformed functions $\hat{\psi}_{i}$ and $\hat{\dot{G}}^{\ell}$, defined on the unit interval, is $\zeta$, whereas $\xi$ denotes the argument of the functions $\psi_{i}$ and $\dot{G}$, defined on the element $E^{\ell}$.

The functions $\hat{\psi}_{i}, \ldots, \hat{\psi}_{i+p}$ are NURBS basis functions of degree $p$, whose support includes the knot span $[0,1]$. The additional $2 p$ knots needed to define these NURBS basis functions are contained in the interval $[-p K,+p K+1]$, where the constant $K$ was introduced in Assumption 1. 


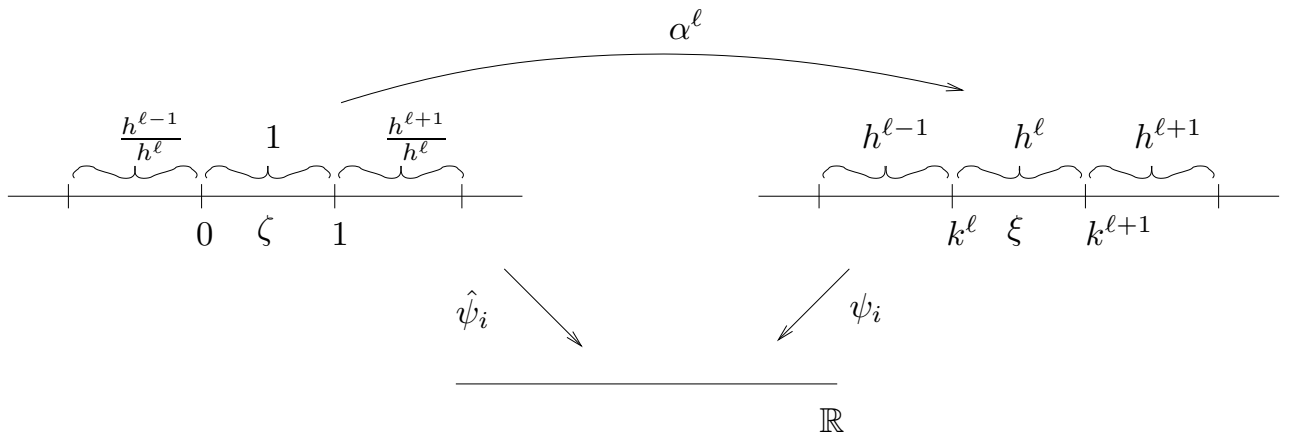

Figure 5: The mapping $\alpha^{\ell}: \mathbb{R} \rightarrow \mathbb{R}$, which maps the unit element $[0,1]$ to the element $E^{\ell}=\left[k^{\ell}, k^{\ell+1}\right]$. The function $\hat{\psi}_{i}:[0,1] \rightarrow \mathbb{R}$ is given by $\hat{\psi}_{i}=\psi_{i} \circ \alpha^{\ell}$.

In addition, according to Assumption 2, the weights used to define these functions belong to the interval $[-1 / W, W]$. Consequently, given an element $E^{\ell}$, the functions $\hat{\psi}_{j}$, whose support includes the transformed element $[0,1]$, depend on parameters (the knots and weights) which vary within a bounded and closed set. This observation will be exploited in the following sections to bound the eigenvalues of the element stiffness and mass matrices.

\subsection{The maximum eigenvalue of the element stiffness matrix}

First we consider the maximum eigenvalue of the element stiffness matrix, which is needed to bound the numerator of the condition number. We obtain the following result:

Lemma 15 There exists a positive constant $\bar{\lambda}$, which depends solely on $K, W$ and $p$, such that the maximum eigenvalue of the element stiffness matrix satisfies

$$
\lambda_{\max }\left(\mathbf{S}^{\ell}\right) \leq \frac{1}{h^{\ell} \omega_{\min }^{\ell}} \bar{\lambda}
$$

Proof. We consider an entry (15) of the element stiffness matrix. By factoring out the term

$$
\max _{\xi \in E^{\ell}} \frac{1}{\omega(\xi)}=\frac{1}{\min _{\xi \in E^{\ell}} \omega(\xi)}=\frac{1}{\omega_{\min }^{\ell}},
$$

it can be rewritten as

$$
s_{i, j}^{\ell}=\frac{1}{\omega_{\min }^{\ell}} \int_{k^{\ell}}^{k^{\ell+1}} \dot{\psi}_{i}(\xi) \dot{\psi}_{j}(\xi) \frac{\omega_{\min }^{\ell}}{\omega(\xi)} \mathrm{d} \xi .
$$


We transform the integrals from the knot $\operatorname{span}\left[k^{\ell}, k^{\ell+1}\right]$ to the interval $[0,1]$. The derivatives of the NURBS basis functions satisfy

$$
\hat{\dot{\psi}}_{i}=\left(\frac{\partial}{\partial \xi} \psi_{i}\right) \circ \alpha^{\ell}=\frac{\partial}{\partial \zeta}\left(\psi_{i} \circ \alpha^{\ell}\right) \frac{1}{h^{\ell}}=\frac{\partial}{\partial \zeta} \hat{\psi}_{i} \frac{1}{h^{\ell}}=\dot{\hat{\psi}}_{i} \frac{1}{h^{\ell}} .
$$

Note that $\hat{\dot{\psi}}_{i}$ are the transformations of the derivatives, whereas $\dot{\hat{\psi}}_{i}$ are the derivatives of the transformed functions. Taking $\mathrm{d} \xi=h^{\ell} \mathrm{d} \zeta$ into account, we rewrite the element stiffness matrix as

$$
\mathbf{S}^{\ell}=\frac{1}{h^{\ell} \omega_{\min }^{\ell}} \hat{\mathbf{S}}^{\ell}
$$

where the entries $\hat{s}_{i, j}^{\ell}$ of the normalized element stiffness matrix $\hat{\mathbf{S}}^{\ell}$ are

$$
\hat{s}_{i, j}^{\ell}=\int_{0}^{1} \dot{\hat{\psi}}_{i}(\zeta) \dot{\hat{\psi}}_{j}(\zeta) \underbrace{\frac{\omega_{\min }^{\ell}}{\hat{\omega}(\zeta)}}_{(\star)} \mathrm{d} \zeta .
$$

These entries depend on the derivatives $\dot{\hat{\psi}}_{i}$ of NURBS basis functions which are determined by parameters from a bounded set of knots and weights, as described in Section 4.1, and on the weight function $(\star)$, which varies in the interval $[0,1]$. Consequently, the normalized element stiffness matrices belong to a bounded set of symmetric matrices, whose bounds depend on the constants $K$ and $W$ and the polynomial degree $p$ of the basis functions. We can therefore use Lemma 4 to bound the matrix from above,

$$
\hat{\mathbf{S}}^{\ell} \leq \bar{\lambda} \mathbf{I}, \quad \text { or, equivalently, } \quad \lambda_{\max }\left(\hat{\mathbf{S}}^{\ell}\right) \leq \bar{\lambda}
$$

with a strictly positive constant $\bar{\lambda}$ which depends solely on $K, W$ and $p$. The proof is completed by combining this observation with (17).

\subsection{The minimum eigenvalue of the element mass matrix}

We consider the minimum eigenvalue of the element mass matrix in order to bound the denominator of the condition number via the Friedrichs inequality. The bound depends on the polynomial degree $p$ and the constants $K$ and $W$, see Assumptions 1 and 2 .

Lemma 16 There exists a positive constant $\underline{\lambda}$, which depends solely on $K, W$ and $p$ such that the minimum eigenvalue of the element mass matrix satisfies

$$
\lambda_{\min }\left(\mathbf{M}^{\ell}\right) \geq h^{\ell} \omega_{\min }^{\ell} \underline{\lambda} .
$$


Proof. We consider an entry (15) of the element mass matrix. By factoring out the term $\omega_{\max }^{\ell}$ and transforming the integrals to the unit interval, it can be rewritten as

$$
m_{i, j}^{\ell}=\omega_{\max }^{\ell} \int_{k^{\ell}}^{k^{\ell+1}} \psi_{i}(\xi) \psi_{j}(\xi) \frac{\omega(\xi)}{\omega_{\max }^{\ell}} \mathrm{d} \xi=h^{\ell} \omega_{\max }^{\ell} \underbrace{\int_{0}^{1} \hat{\psi}_{i}(\zeta) \hat{\psi}_{j}(\zeta) \frac{\hat{\omega}(\zeta)}{\omega_{\max }^{\ell}} \mathrm{d} \zeta}_{=\hat{m}_{i . j}^{\ell}} .
$$

We use the integrals $\hat{m}_{i, j}^{\ell}$ in the rightmost term to define the normalized element mass matrix $\hat{\mathbf{M}}^{\ell}$. Clearly, we have $\mathbf{M}^{\ell}=h^{\ell} \omega_{\max }^{\ell} \hat{\mathbf{M}}^{\ell}$.

In addition we consider simplified normalized element mass matrices $\hat{\hat{\mathbf{M}}}^{\ell}$ with the entries

$$
\hat{\hat{m}}_{i, j}^{\ell}=\int_{0}^{1} \hat{\psi}_{i}(\zeta) \hat{\psi}_{j}(\zeta) \mathrm{d} \zeta
$$

which are guaranteed to be positive definite, since the NURBS basis functions $\hat{\psi}_{i}$ on each knot span are linearly independent. The knots and weights which determine these functions vary within a closed and bounded set, as described in Section 4.1. Thus, the simplified element mass matrices belong to a closed and bounded set of symmetric and positive definite matrices, whose bounds depend on the constants $K$ and $W$ and the polynomial degree $p$. We use the second part of Lemma 4 to bound these matrices from below,

$$
\hat{\hat{\mathbf{M}}}^{\ell} \geq \underline{\lambda} \mathbf{I},
$$

where the positive constant $\underline{\lambda}$ depends solely on $K, W$ and $p$.

On the other hand, Assumption 9 guarantees

$$
\frac{\hat{\omega}(\zeta)}{\omega_{\max }^{\ell}} \geq \delta^{\ell}
$$

since the ranges of $\omega=|\dot{G}|$ on $\left[k^{\ell}, k^{\ell+1}\right]$ and $\hat{\omega}=|\hat{\dot{G}}|$ on $[0,1]$ are identical. By swapping summation (which arises due to matrix-vector multiplication) and integration, a short computation confirms that

$$
\mathbf{c}^{T} \hat{\mathbf{M}}^{\ell} \mathbf{c}=\int_{0}^{1}\left(\sum_{i} c_{i} \hat{\psi}_{i}(\zeta)\right)^{2} \frac{\hat{\omega}(\zeta)}{\omega_{\max }^{\ell}} \mathrm{d} \zeta \geq \delta^{\ell} \int_{0}^{1}\left(\sum_{i} c_{i} \hat{\psi}_{i}(\zeta)\right)^{2} \mathrm{~d} \zeta=\delta^{\ell} \mathbf{c}^{T} \hat{\hat{\mathbf{M}}}^{\ell} \mathbf{c}
$$

holds for all vectors c. This implies

$$
\hat{\mathbf{M}}^{\ell} \geq \delta^{\ell} \hat{\hat{\mathbf{M}}}^{\ell}
$$

Using the definition $\delta^{\ell}=\frac{\omega_{\min }^{\ell}}{\omega_{\max }^{\ell}}$, the lower bound (18) follows from (19), (20) and (21). 


\subsection{The bound of the condition number}

The previous observations and Assumptions 1, 2 and 9 lead to the following Theorem:

Theorem 17. Let $L$ denote the length of the physical domain. The condition number of the stiffness matrix is bounded by

$$
\kappa(\mathbf{S}) \leq C L^{2} \frac{1}{\min _{\ell}\left(h^{\ell} \omega_{\min }^{\ell}\right)^{2}},
$$

where the constant $C$ depends solely on $K, W$ and $p$.

Proof. This bound is obtained by the general result of Theorem 14 with the particular bounds for the eigenvalues of the element stiffness and mass matrices, which were presented in Lemmas 15 and 16. The ratio of the two constants $\bar{\lambda} / \underline{\lambda}$ multiplied with the factor $(p+1)$, which is inherited from the estimate of the global stiffness matrix by element stiffness matrices, defines another constant $C$, which depends on $K, W$ and $p$. The diameter $\operatorname{diam}(\Omega)$ of the physical domain is given by the length $L$ of the physical domain.

Remark 18 The constant $C$ grows monotonically with increasing values of $K$ and $W$ since the associated bounded sets of symmetric matrices grow when enlarging these values. When considering different parameterizations of a given computational domain, the constants $C$ are generally different, since the values of $K$ and $W$ might be different. The constants $K$ and $W$ depend on the knots and on the weights. If the knots and the weights are fixed, the constants $K$ and $W$ do not depend on the location of the control points. For small variations of knots and weights, the constants $C$ are in the same order of magnitude.

The result for the bound of the condition number admits a geometric interpretation. We denote with $\omega_{\text {avg }}^{\ell}$ the average value of $\omega=|\dot{G}|$ in the element $E^{\ell}$, i.e.,

$$
\omega_{\mathrm{avg}}^{\ell}=\frac{1}{h^{\ell}} \int_{k^{\ell}}^{k^{\ell+1}}|\dot{G}(\xi)| \mathrm{d} \xi=\frac{1}{h^{\ell}}\left|G\left(k^{\ell+1}\right)-G\left(k^{\ell}\right)\right|=\frac{1}{h^{\ell}} h_{\mathrm{phys}}^{\ell},
$$

where $h_{\mathrm{phys}}^{\ell}$ denotes the length of the knot span in the physical domain. Thus we have $h^{\ell} \omega_{\mathrm{avg}}^{\ell}=h_{\mathrm{phys}}^{\ell}$. For sufficiently fine discretizations, the value of $\dot{G}$ is nearly constant in the element $\left[k^{\ell}, k^{\ell+1}\right]$, thus

$$
\omega_{\min }^{\ell} \approx \omega_{\max }^{\ell} \approx \omega_{\mathrm{avg}}^{\ell}
$$


Hence we can approximate the bound for the condition number by

$$
C L^{2} \frac{1}{\min _{\ell}\left(h_{\mathrm{phys}}^{\ell}\right)^{2}},
$$

where the constant $C$ depends solely on $K, W$ and $p$. This expression indicates that the smallest value of the bound is obtained by a uniform distribution of the knot spans in the physical domain. We shall reconsider this observation in Example 2 (see Section 7).

Both the condition number and its bound are invariant under uniform scaling and translations. We consider the case of scaling.

If the physical domain is scaled by a factor of $f$ (which is realized by multiplying $G$ with $f$ ), then both the minimum and the maximum eigenvalue of the stiffness matrix $\mathbf{S}$ are scaled by $1 / f$. Consequently, the condition number remains unchanged.

Now we consider the various quantities in the bound. The length $L$ of the domain is scaled by $f$, and so is $\omega=|\dot{G}|$. All other quantities remain unchanged. Thus, the bound is independent of $f$.

\section{The case $D=2$}

We proceed similarly to the one-dimensional case. After transforming the integrals to the unit square, we derive the bounds for the eigenvalues of the element stiffness and mass matrices. This allows us to formulate a bound for the condition number of the global stiffness matrix.

\subsection{Transformation to the unit square}

Each element $E^{\ell}$ is the Cartesian product of two intervals,

$$
E^{\ell}=\left[k_{1}^{\ell_{1}}, k_{1}^{\ell_{1}+1}\right] \times\left[k_{2}^{\ell_{2}}, k_{2}^{\ell_{2}+1}\right] .
$$

It is an axis-aligned box in the parameter domain with edges of lengths $h_{1}^{\ell_{1}}$ and $h_{2}^{\ell_{2}}$, both of which are non-zero for non-empty elements. For later reference we introduce the following abbreviation:

$$
H_{2}^{\ell}=\frac{\left(h_{1}^{\ell_{1}}\right)^{2}+\left(h_{2}^{\ell_{2}}\right)^{2}}{h_{1}^{\ell_{1}} h_{2}^{\ell_{2}}}
$$

Note that $\boldsymbol{\ell}=\left(\ell_{1}, \ell_{2}\right)$ is a multi-index which identifies the elements. 
The geometry mapping $\mathbf{G}$ is a vector-valued function. The matrix $\mathbf{N}$ used to transform the integrals from the physical domain to the parameter domain is a symmetric and positive definite $2 \times 2$ matrix, see (6). Its entries will be denoted by

$$
\mathbf{N}=\left(\begin{array}{ll}
N_{1,1} & N_{1,2} \\
N_{2,1} & N_{2,2}
\end{array}\right), \text { with } N_{1,2}=N_{2,1}
$$

The element stiffness and mass matrices $\mathbf{S}^{\ell}=\left(s_{\mathbf{i}, \mathbf{j}}^{\ell}\right)_{\mathbf{i}, \mathbf{j}}$ and $\mathbf{M}^{\ell}=\left(m_{\mathbf{i}, \mathbf{j}}^{\ell}\right)_{\mathbf{i}, \mathbf{j}}$ have the entries

$$
s_{\mathbf{i}, \mathbf{j}}^{\ell}=\int_{E^{\ell}} \nabla \psi_{\mathbf{i}}^{T}(\boldsymbol{\xi}) \mathbf{N}(\boldsymbol{\xi}) \nabla \psi_{\mathbf{j}}(\boldsymbol{\xi}) \mathrm{d} \boldsymbol{\xi} \quad \text { and } \quad m_{\mathbf{i}, \mathbf{j}}^{\ell}=\int_{E^{\ell}} \psi_{\mathbf{i}}(\boldsymbol{\xi}) \psi_{\mathbf{j}}(\boldsymbol{\xi}) \omega(\boldsymbol{\xi}) \mathrm{d} \boldsymbol{\xi},
$$

where we use multi-indices $\mathbf{i}=\left(i_{1}, i_{2}\right)$ and $\mathbf{j}=\left(j_{1}, j_{2}\right)$ to identify the NURBS basis functions.

In the sequel we will transform the integrals from an element $E^{\ell}$ to the unit element $[0,1]^{2}$. We will denote the transformed functions defined on the unit element by

$$
\hat{\psi}_{\mathbf{i}}=\psi_{\mathbf{i}} \circ \alpha^{\ell}, \quad \hat{\mathbf{N}}^{\ell}=\mathbf{N} \circ \alpha^{\ell}, \quad \hat{\mathbf{J}}^{\ell}=\mathbf{J} \circ \alpha^{\ell},
$$

etc., where $\alpha^{\ell}$ is the affine mapping

$$
\alpha^{\ell}: \mathbb{R}^{2} \rightarrow \mathbb{R}^{2}: \boldsymbol{\zeta}=\left(\zeta_{1}, \zeta_{2}\right) \mapsto \boldsymbol{\xi}(\boldsymbol{\zeta})=\left(h_{1}^{\ell_{1}} \zeta_{1}+k_{1}^{\ell_{1}}, h_{2}^{\ell_{2}} \zeta_{2}+k_{2}^{\ell_{2}}\right),
$$

which satisfies $[0,1]^{2} \rightarrow E^{\ell}$. Clearly, the transformed functions depend on the element index $\ell$ also. This dependency, however, is omitted in the notation in order to keep it as simple as possible.

\subsection{The maximum eigenvalue of the element stiffness matrix}

Lemma 19 The maximum eigenvalue of the element stiffness matrix is bounded from above by

$$
\lambda_{\max }\left(\mathbf{S}^{\ell}\right) \leq \lambda_{\max }\left(\mathbf{N}_{\max }^{\ell}\right) H_{2}^{\ell} \bar{\lambda}
$$

where $H_{2}^{\ell}$ is defined in Eq. (23) and the strictly positive constant $\bar{\lambda}$ depends solely on $K, W$ and the polynomial degrees $p_{1}$ and $p_{2}$.

Proof. We denote the derivative of $\psi_{\mathbf{i}}$ with respect to $\xi_{k}$ by $\psi_{\mathbf{i} \mid k}=\left(\partial \psi_{\mathbf{i}}\right) /\left(\partial \xi_{k}\right)$. The element stiffness matrix (24) can be represented as a sum of four matrices,

$$
\mathbf{S}^{\ell}=\mathbf{A}_{1,1}^{\ell}+\mathbf{A}_{1,2}^{\ell}+\mathbf{A}_{2,1}^{\ell}+\mathbf{A}_{2,2}^{\ell},
$$


which correspond to the four entries of the matrix $\mathbf{N}$, i.e.,

$$
\mathbf{A}_{\alpha, \beta}^{\boldsymbol{\ell}}=\left(a_{\alpha, \beta, \mathbf{i}, \mathbf{j}}^{\boldsymbol{\ell}}\right)_{\mathbf{i}, \mathbf{j}} \quad \text { with } \quad a_{\alpha, \beta, \mathbf{i}, \mathbf{j}}^{\boldsymbol{\ell}}=\int_{E^{\ell}} \psi_{\mathbf{i} \mid \alpha}(\boldsymbol{\xi}) \psi_{\mathbf{j} \mid \beta}(\boldsymbol{\xi}) N_{\alpha, \beta}(\boldsymbol{\xi}) \mathrm{d} \boldsymbol{\xi},
$$

where the indices $\alpha, \beta$ take the values 1 and 2. For each of these matrices we define an associated normalized version by factoring out $N_{\alpha, \beta, \max }^{\ell}$ and transforming the integral to the unit square,

$$
\hat{\mathbf{A}}_{\alpha, \beta}^{\ell}=\left(\hat{a}_{\alpha, \beta, \mathbf{i}, \mathbf{j}}^{\ell}\right)_{\mathbf{i}, \mathbf{j}} \quad \text { with } \quad \hat{a}_{\alpha, \beta, \mathbf{i}, \mathbf{j}}^{\ell}=\int_{[0,1]^{2}} \hat{\psi}_{\mathbf{i} \mid \alpha}(\boldsymbol{\zeta}) \hat{\psi}_{\mathbf{j} \mid \beta}(\boldsymbol{\zeta}) \underbrace{\frac{\hat{N}_{\alpha, \beta}(\boldsymbol{\zeta})}{N_{\alpha, \beta, \max }^{\ell}}}_{(\star)} \mathrm{d} \boldsymbol{\zeta} .
$$

We note that the term marked with $(\star)$ is bounded in $[-1,1]$.

The transformation to the unit square introduces the factor $h_{1}^{\ell_{1}} h_{2}^{\ell_{2}}$, while the transformation of the derivative is governed by the chain rule

$$
\frac{\partial}{\partial \xi_{i}}=\frac{1}{h_{i}^{\ell_{i}}} \frac{\partial}{\partial \zeta_{i}}
$$

Consequently, the element stiffness matrix is a weighted sum of the four normalized matrices,

$$
\mathbf{S}^{\ell}=N_{1,1, \max }^{\ell} \frac{h_{2}^{\ell_{2}}}{h_{1}^{\ell_{1}}} \hat{\mathbf{A}}_{1,1}^{\ell}+N_{1,2, \max }^{\ell} \hat{\mathbf{A}}_{1,2}^{\ell}+N_{1,2, \max }^{\ell} \hat{\mathbf{A}}_{2,1}^{\ell}+N_{2,2, \max }^{\ell} \frac{h_{1}^{\ell_{1}}}{h_{2}^{\ell_{2}}} \hat{\mathbf{A}}_{2,2}^{\ell} .
$$

The entries of the normalized matrices $\hat{\mathbf{A}}_{\alpha, \beta}^{\ell}$ are determined by derivatives $\hat{\psi}_{\boldsymbol{i} \mid \alpha}$ of tensorproduct NURBS basis functions whose knots and weights vary within a closed and bounded set of parameters (according to Assumptions 1 and 2). Thus, the derivatives are bounded, too. Furthermore, the entries of the normalized matrices depend on bounded functions with values in $[-1,1]$ (marked by $(\star)$ in Eq. (26)). Consequently, all these normalized matrices belong to a bounded set of symmetric matrices. Lemma 4 guarantees the existence of an upper bound

$$
\hat{\mathbf{A}}_{\alpha, \beta}^{\ell} \leq \bar{\lambda} \mathbf{I}
$$

which depends solely on $K, W$ and the polynomial degrees $p_{1}$ and $p_{2}$. We use this observation to bound the largest eigenvalue of the element stiffness matrix

$$
\lambda_{\max }\left(\mathbf{S}^{\ell}\right) \leq \bar{\lambda} \underbrace{\left(N_{1,1, \max }^{\ell} \frac{h_{2}^{\ell_{2}}}{h_{1}^{\ell_{1}}}+N_{1,2, \max }^{\ell}+N_{1,2, \max }^{\ell}+N_{2,2, \max }^{\ell} \frac{h_{1}^{\ell_{1}}}{h_{2}^{\ell_{2}}}\right)}_{=B} .
$$


Finally we rewrite and bound the term $B$ on the right-hand side,

$$
B=h_{1}^{\ell_{1}} h_{2}^{\ell_{2}}\left(\begin{array}{c}
1 / h_{1}^{\ell_{1}} \\
1 / h_{2}^{\ell_{2}}
\end{array}\right)^{T} \mathbf{N}_{\max }^{\ell}\left(\begin{array}{c}
1 / h_{1}^{\ell_{1}} \\
1 / h_{2}^{\ell_{2}}
\end{array}\right) \leq \lambda_{\max }\left(\mathbf{N}_{\max }^{\ell}\right) \underbrace{h_{1}^{\ell_{1}} h_{2}^{\ell_{2}}\left(\begin{array}{c}
1 / h_{1}^{\ell_{1}} \\
1 / h_{2}^{\ell_{2}}
\end{array}\right)^{T}\left(\begin{array}{c}
1 / h_{1}^{\ell_{1}} \\
1 / h_{2}^{\ell_{2}}
\end{array}\right)}_{=H_{2}^{\ell}}
$$

based on the inequality

$$
\mathbf{x}^{T} \mathbf{N} \mathbf{x} \leq \lambda_{\max }(\mathbf{N}) \mathbf{x}^{T} \mathbf{x}
$$

which is valid for any real quadratic matrix $\mathbf{N} \in \mathbb{R}^{n \times n}$ and any vector $\mathbf{x} \in \mathbb{R}^{n}$.

\subsection{The minimum eigenvalue of the element mass matrix}

Again we use Assumptions 1, 2 and 9 in order to obtain the second bound.

Lemma 20 There exists a positive constant $\underline{\lambda}$, which depends solely on $K, W$ and the polynomial degrees $p_{1}$ and $p_{2}$, such that the minimum eigenvalue of the element mass matrix satisfies

$$
\lambda_{\min }\left(\mathbf{M}^{\ell}\right) \geq h_{1}^{\ell_{1}} h_{2}^{\ell_{2}} \omega_{\min }^{\ell} \underline{\lambda} .
$$

Proof. The proof is analogous to the case $D=1$. The only difference appears when transforming the integrals to the unit square, where the original element mass matrix and the normalized one are now related by $\mathbf{M}^{\ell}=h_{1}^{\ell_{1}} h_{2}^{\ell_{2}} \omega_{\max }^{\ell} \hat{\mathbf{M}}^{\ell}$, due to the bivariate integration.

\subsection{The bound of the condition number}

The previous two Lemmas and Theorem 14 imply the following result:

Theorem 21. Let $h_{1}^{\ell_{1}}$ and $h_{2}^{\ell_{2}}$ denote the lengths of the knot spans in the two parameter directions in the parameter domain. The condition number of the stiffness matrix is bounded by

$$
\kappa(\mathbf{S}) \leq C(\operatorname{diam}(\Omega))^{2} \frac{\max _{\ell}\left(\lambda_{\max }\left(\mathbf{N}_{\max }^{\ell}\right) H_{2}^{\ell}\right)}{\min _{\ell}\left(h_{1}^{\ell_{1}} h_{2}^{\ell_{2}} \omega_{\min }^{\ell}\right)},
$$

where $H_{2}^{\ell}$ is defined in Eq. (23) and the constant $C$ depends solely on $K, W$ and the polynomial degrees $p_{1}$ and $p_{2}$. 
Note that the constants $\bar{\lambda}, \underline{\lambda}$ and the factor $\left(\prod_{d=1}^{2}\left(p_{d}+1\right)\right)$ are combined to the constant $C$. Similar to the observation in Remark 18, the constant $C$ can be shown to grow monotonically with increasing values of $K$ and $W$, since the associated bounded sets of symmetric matrices grow when enlarging these values.

We can further split the expression in Eq. (28) to separate the terms depending on the geometry mapping from those that depend on the length of the knot spans,

$$
\kappa(\mathbf{S}) \leq C(\operatorname{diam}(\Omega))^{2} \frac{\max _{\ell}\left(\lambda_{\max }\left(\mathbf{N}_{\max }^{\ell}\right)\right)}{\min _{\ell} \omega_{\min }^{\ell}} \frac{\max _{\ell} H_{2}^{\ell}}{\min _{\ell}\left(h_{1}^{\ell_{1}} h_{2}^{\ell_{2}}\right)} .
$$

Again, the bound can be interpreted in a geometric way. The matrix $\mathbf{N}$ can be expressed as

$$
\mathbf{N}=\frac{1}{\omega}\left(\begin{array}{cc}
\left\|\mathbf{G}_{\mid 2}\right\|^{2} & -\mathbf{G}_{\mid 1} \cdot \mathbf{G}_{\mid 2} \\
-\mathbf{G}_{\mid 1} \cdot \mathbf{G}_{\mid 2} & \left\|\mathbf{G}_{\mid 1}\right\|^{2}
\end{array}\right),
$$

where $\mathbf{G}_{\mid i}$ denotes the partial derivative of the geometry mapping with respect to $\xi_{i}$. For a general $2 \times 2$ matrix $\mathbf{A}$ with

$$
\mathbf{A}=\left(\begin{array}{ll}
a_{1,1} & a_{1,2} \\
a_{2,1} & a_{2,2}
\end{array}\right)
$$

the maximum eigenvalue is given by

$$
\lambda_{\max }(\mathbf{A})=\frac{1}{2}\left(a_{1,1}+a_{2,2}+\sqrt{\left(a_{1,1}-a_{2,2}\right)^{2}+4 a_{1,2} a_{2,1}}\right) .
$$

Using this closed-form representation we can rewrite $\lambda_{\max }\left(\mathbf{N}_{\max }^{\ell}\right) \approx \max _{\boldsymbol{\xi} \in E^{\ell}} \frac{\bar{G}(\boldsymbol{\xi})}{\omega(\boldsymbol{\xi})}$ with

$$
\bar{G}(\boldsymbol{\xi})=\frac{1}{2}\left(\left\|\mathbf{G}_{\mid 1}\right\|^{2}+\left\|\mathbf{G}_{\mid 2}\right\|^{2}+\left(\left(\left\|\mathbf{G}_{\mid 1}\right\|^{2}-\left\|\mathbf{G}_{\mid 2}\right\|^{2}\right)^{2}+4 \cos ^{2}(\alpha)\left\|\mathbf{G}_{\mid 1}\right\|^{2}\left\|\mathbf{G}_{\mid 2}\right\|^{2}\right)^{\frac{1}{2}}\right),
$$

where $\alpha$ represents the angle between the parameter lines. Similar to the case $D=1$ we define the average value of $\omega$ in the element $E^{\ell}$ by

$$
\omega_{\mathrm{avg}}^{\ell}=\frac{1}{h_{1}^{\ell_{1}} h_{2}^{\ell_{2}}} \int_{E^{\ell}} \omega(\boldsymbol{\xi}) \mathrm{d} \boldsymbol{\xi}=\frac{1}{h_{1}^{\ell_{1}}} \frac{1}{h_{2}^{\ell_{2}}} A^{\ell},
$$

where $A^{\ell}$ denotes the area of the element $E^{\ell}$ in the physical domain. Thus we have $h_{1}^{\ell_{1}} h_{2}^{\ell_{2}} \omega_{\mathrm{avg}}^{\ell}=A^{\ell}$.

Consider the case of a very fine isogeometric discretization, such that the various quantities in our bound do not vary significantly within the elements, and hence also $\omega_{\min }^{\ell} \approx \omega_{\text {avg }}^{\ell}$. 
Then the bound in Eq. (28) can be approximated by

$$
C(\operatorname{diam}(\Omega))^{2} \frac{\max _{\ell, \boldsymbol{\xi}} \bar{G}(\boldsymbol{\xi})\left(\left(h_{1}^{\ell_{1}}\right)^{2}+\left(h_{2}^{\ell_{2}}\right)^{2}\right)}{\min _{\ell}\left(A^{\ell}\right)^{2}} .
$$

In order to obtain a small bound, the lengths of both partial derivatives should be close to equal and the angle between them should be close to orthogonal. Furthermore, elements with a small area should be avoided, i.e., the area should not vary too much between elements in the physical domain.

Both the condition number and its bound are invariant under uniform scaling, translations and rotations, i.e., under Euclidean similarities. We consider the case of scaling.

If the physical domain is scaled by a factor of $f$ (which is realized by multiplying $\mathbf{G}$ with $f$ ), then both the minimum and the maximum eigenvalue of the stiffness matrix $\mathbf{S}$ remain unchanged and hence the condition number remains unchanged.

Now we consider the various quantities in the bound. The diameter of the domain is scaled by $f$, but $\omega=|\operatorname{det} \mathbf{J}|$ is scaled by $f^{2}$. All other quantities remain unchanged. Thus, the bound is independent of $f$.

\section{The case $D=3$}

The derivation of the bound for the condition number is analogous to the two-dimensional case. We give only the main result, where we need the expression

$$
H_{3}^{\ell}=\frac{\left(h_{2}^{\ell_{2}}\right)^{2}\left(h_{3}^{\ell_{3}}\right)^{2}+\left(h_{1}^{\ell_{1}}\right)^{2}\left(h_{3}^{\ell_{3}}\right)^{2}+\left(h_{1}^{\ell_{1}}\right)^{2}\left(h_{2}^{\ell_{2}}\right)^{2}}{h_{1}^{\ell_{1}} h_{2}^{\ell_{2}} h_{3}^{\ell_{3}}},
$$

which involves lengths of knot spans $h_{i}^{\ell_{i}}, i=1,2,3$.

Theorem 22. Let $h_{1}^{\ell_{1}}, h_{2}^{\ell_{2}}$ and $h_{3}^{\ell_{3}}$ denote the lengths of the knot spans in the three parameter directions in the parameter domain. The condition number of the stiffness matrix is bounded by

$$
\kappa(\mathbf{S}) \leq C(\operatorname{diam}(\Omega))^{2} \frac{\max _{\ell}\left(\lambda_{\max }\left(\mathbf{N}_{\max }^{\ell}\right) H_{3}^{\ell}\right)}{\min _{\ell}\left(h_{1}^{\ell_{1}} h_{2}^{\ell_{2}} h_{3}^{\ell_{3}} \omega_{\min }^{\ell}\right)},
$$

where $H_{3}^{\ell}$ is defined in Eq. (30) and the constant $C$ depends solely on $K, W$ and the polynomial degrees $p_{i}, i=1,2,3$ of $B$-spline/NURBS basis functions in the three parameter directions. 
We can further split the bound in Eq. (31) to separate the term which depends on the geometry mapping from the term which quantifies the influence of the knot spans,

$$
\kappa(\mathbf{S}) \leq C(\operatorname{diam}(\Omega))^{2} \frac{\max _{\ell} \lambda_{\max }\left(\mathbf{N}_{\max }^{\ell}\right)}{\min _{\ell} \omega_{\min }^{\ell}} \frac{\max _{\ell} H_{3}^{\ell}}{\min _{\ell}\left(h_{1}^{\ell_{1}} h_{2}^{\ell_{2}} h_{3}^{\ell_{3}}\right)} .
$$

In the case $D=3$ we cannot derive a simple closed formula for the maximum eigenvalue of the matrix $\mathbf{N}_{\max }^{\ell}$ and thus it is difficult to derive a geometric interpretation. One may guess though that the findings of the case $D=2$ (orthogonal parameter directions, similar lengths of edges in the physical domain) apply to this situation, too. Furthermore, for fine discretizations, the expression $\omega_{\min }^{\ell} h_{1}^{\ell_{1}} h_{2}^{\ell_{2}} h_{3}^{\ell_{3}}$, appearing in the denominator of the bound, approximates the volume of an element in the physical domain. Hence, we obtain a small bound if the volumes of the elements in the physical domain do not vary too much.

Both the condition number and its bound are invariant under uniform scaling, translations and rotations, i.e., under Euclidean similarities. We consider the case of scaling by a factor $f$. Applying this scaling, both the maximum and the minimum eigenvalue of the global stiffness matrix $\mathbf{S}$ are multiplied by a factor of $f$, hence the condition number remains unchanged. Considering the quantities in the bound, we obtain that the diameter of the domain is scaled by $f$. The term $\omega=|\operatorname{det} \mathbf{J}|$ is scaled by $f^{3}$. The matrix $\mathbf{N}$ and its eigenvalues are now scaled by $f$. Putting things together, it is confirmed that the bound is invariant under uniform scaling.

\section{Examples}

\section{Example 1: Asymptotic behavior}

The first example illustrates the fact that the bounds for the condition number derived in the previous sections are asymptotically optimal.

The case $D=1$. We consider a B-spline parameterization of the unit interval. We use uniform knot vectors and the identity as geometry mapping, thus $K=W=1, \omega=1$ and therefore also $\omega_{\min }^{\ell}=\omega_{\max }^{\ell}=1$. We obtain the bound

$$
\kappa(\mathbf{S}) \leq C L^{2} \frac{1}{\min _{\ell}\left(h^{\ell} \omega_{\min }^{\ell}\right)^{2}}=C L^{2} \frac{1}{h^{2}} .
$$

In Table 2 we analyze the behavior of the condition number and its bound if we apply uniform $h$-refinement. We consider basis functions of degree $p=2$ and of degree $p=3$. 
We display the condition number $\kappa(\mathbf{S})$, the computed bound and the ratios of these values of consecutive refinement levels. For the sake of brevity we display only the coefficients of the bounds in the table. Each coefficient is multiplied by $C$, which depends on $K, W$ and $p$. We obtain that the ratio of condition numbers of two consecutive refinement levels converges to 4 . The same behavior can be found for the bound. Hence, if we apply uniform $h$-refinement, then the condition number and its bound scale as $h^{-2}$. The behavior of the condition number of the stiffness matrix for IGA has also been studied recently, see, e.g., $[36]$.

\begin{tabular}{cc|cc|cc|cc}
$\begin{array}{c}\text { refinement } \\
\text { level }\end{array}$ & $h$ & $\kappa(\mathbf{S})$ & $\begin{array}{c}|r| \\
\text { ratio to } \\
\text { previous } \\
\text { level }\end{array}$ & $\kappa(\mathbf{S})$ & $\begin{array}{c}|c| \\
\text { ratio to } \\
\text { previous } \\
\text { level }\end{array}$ & $\begin{array}{c}\text { ratio to } \\
\text { bound } \\
\text { previous } \\
\text { level }\end{array}$ \\
\hline 0 & 1 & 1.00 & & 1.66 & & 1 & \\
1 & $\frac{1}{2}$ & 1.00 & 1.00 & 3.38 & 2.03 & $2^{2}$ & 4 \\
2 & $\frac{1}{4}$ & 2.78 & 2.78 & 3.51 & 1.04 & $2^{4}$ & 4 \\
3 & $\frac{1}{8}$ & 10.05 & 3.61 & 10.86 & 3.09 & $2^{6}$ & 4 \\
4 & $\frac{1}{16}$ & 39.23 & 3.90 & 41.43 & 3.81 & $2^{8}$ & 4 \\
5 & $\frac{1}{32}$ & 155.95 & 3.98 & 163.96 & 3.96 & $2^{10}$ & 4 \\
6 & $\frac{1}{64}$ & 622.84 & 3.99 & 654.18 & 3.99 & $2^{12}$ & 4 \\
7 & $\frac{1}{128}$ & 2490.39 & 3.99 & 2615.11 & 3.99 & $2^{14}$ & 4
\end{tabular}

Table 2: Condition number, bound (without the factor $C$ ) and ratios to the previous level for different refinement levels using quadratic $(p=2)$ or cubic $(p=3)$ basis functions.

The case $D=2$. A similar asymptotic behavior can also be observed in higher dimensions and also for different parameterizations than the identity mapping, or if the refinement algorithm is just applied in one parameter direction. As an example we consider a parameterization of a quarter annulus with knot vectors

$$
[0,0,0,0.5,1,1,1] \times[0,0,0,1,1,1]
$$

for the initial parameterization. We apply a uniform dyadic knot refinement several times. In Table 3 we display the condition number $\kappa(\mathbf{S})$, the computed bound and the ratios of these values of consecutive refinement levels. For the sake of brevity we display only the 
coefficients of the bounds in the table. Each coefficient is multiplied by $C$, which depends on $K, W, p_{1}$ and $p_{2}$. Since we use quadratic NURBS, we have $p_{1}=p_{2}=2$. We have equal knot spans in both parameter directions, thus we have $K=1$ for all refinement levels. For refinement level 0 , the weights are bounded in $[1 / W, W]$ with $W=\frac{2 \sqrt{2}}{1+\sqrt{2}}$. The constant $W$ to bound the weights converges to 1 as the level increases.

\begin{tabular}{c|cc|cc}
$\begin{array}{c}\text { refinement } \\
\text { level }\end{array}$ & $\kappa(\mathbf{S})$ & $\begin{array}{c}\text { ratio to } \\
\text { previous level }\end{array}$ & bound & $\begin{array}{c}\text { ratio to } \\
\text { previous level }\end{array}$ \\
\hline 0 & 2.93 & & $8.62 \cdot 10^{1}$ & \\
1 & 4.86 & 1.66 & $3.60 \cdot 10^{2}$ & 4.17 \\
2 & 5.63 & 1.16 & $1.47 \cdot 10^{3}$ & 4.08 \\
3 & 10.72 & 1.91 & $5.94 \cdot 10^{3}$ & 4.04 \\
4 & 43.06 & 4.02 & $2.39 \cdot 10^{4}$ & 4.02 \\
5 & 175.35 & 4.07 & $9.57 \cdot 10^{4}$ & 4.01
\end{tabular}

Table 3: Condition number, bound (without the factor $C$ ) and ratios to the previous level for different refinement levels.

This example confirms that the bound is asymptotically optimal since both sequences of ratios converge to 4 as the level of refinement increases.

\section{Example 2 - Compensation of non-uniform knots by the geometry mapping}

Generally, one may expect that the use of highly non-uniform knots leads to large values of the condition number. To some extent, however, the small knot spans can be compensated by a suitable geometry mapping.

The case $D=1$. We consider quadratic B-splines with a knot vector

$$
\left[0,0,0, \frac{1}{2}, \frac{3}{4}, \frac{7}{8}, \frac{15}{16}, \frac{31}{32}, \frac{63}{64}, 1,1,1\right]
$$

see Fig 6 . Note the short knot spans in the right part of the knot vector.

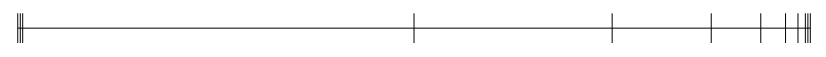

Figure 6: Illustration of the knot vector.

We consider two different parameterizations of the unit interval. On the one hand, we use the identity mapping. On the other hand, we consider the parameterization with 
equidistant control points. For both parameterizations, the constants $p=2, K=4$ and $W=1$ are the same. Thus, the constant $C$ is also the same.

Table 4 displays the condition numbers $\kappa(\mathbf{S})$ and the bounds for the two considered parameterizations. The geometry mapping with equidistant control points instead of the identity mapping leads to a much better condition number and bound.

\begin{tabular}{lcc} 
& identity mapping & equidistant control points \\
\hline$\kappa(\mathbf{S})$ & 43.94 & 10.93 \\
bound & $4.10 \cdot 10^{3}$ & $1.44 \cdot 10^{2}$
\end{tabular}

Table 4: Condition number and bound (without the factor $C$ ) for two parameterizations with non-uniform knots.

We will examine this phenomenon more closely. The plot in the left-hand side of Fig. 7 shows the function $\omega$ for both mappings. For the identity mapping we have $\omega=1$. The mapping with equidistant control points produces a function $\omega$ which reaches large values in regions with short knot spans. However, if we consider $\frac{1}{h^{\ell} \omega}$ instead, which is shown in the right picture, then we observe that it reaches high values for the identity mapping whereas it remains fairly small for the mapping with equidistant control points. Thus, in the case of the mapping with equidistant control points, a small value of $h^{\ell}$ is compensated by a large value of $\omega$, which leads to a more uniform distribution of elements in the physical domain. Thereby this example confirms the geometric interpretation of the bound.

The case $D=2$. The same effect can be observed in the two-dimensional case. We consider different parameterizations of the unit square by quadratic B-splines with the knot vector

$$
\left[0,0,0, \frac{1}{2}, \frac{3}{4}, \frac{7}{8}, \frac{15}{16}, \frac{31}{32}, \frac{63}{64}, \frac{127}{128}, \frac{255}{256}, 1,1,1\right]
$$

in both parameter directions. We compare the identity mapping with a geometry mapping, where the control points are placed in a way such that the elements in the physical domain have equal size and shape. Fig. 8 shows the physical domains with control points and elements for the two analyzed parameterizations.

We compare the condition number and its bound for both parameterizations in Table 5. Again, $C$ depends on the same constants $p_{1}=p_{2}=2, K=4$ and $W=1$. 

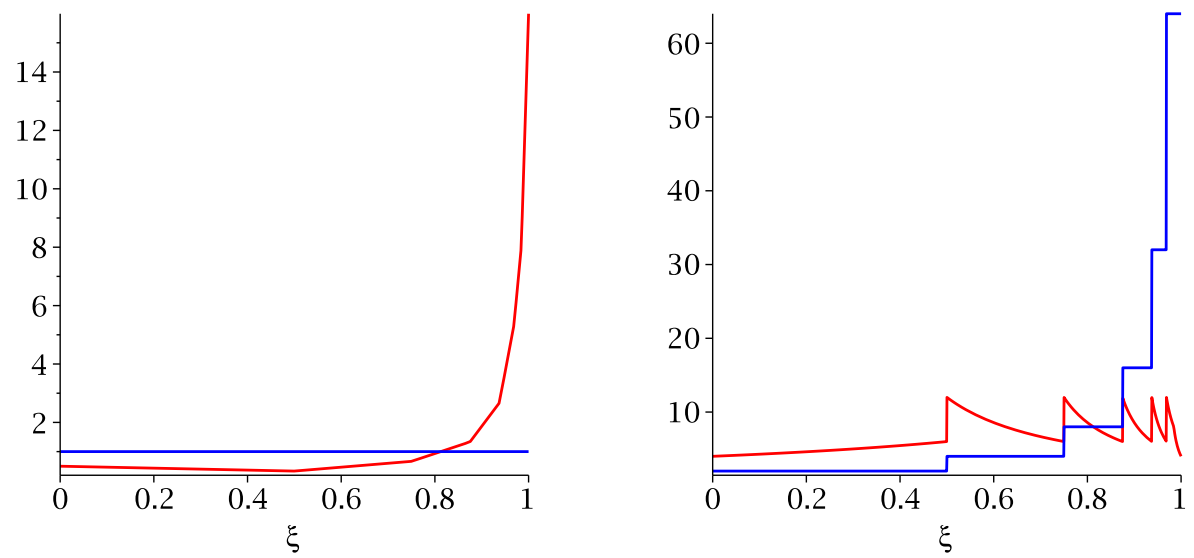

Figure 7: Compensation of a non-uniform knot vector by a suitable geometry mapping: The function $\omega$ (left) and the term $\frac{1}{h^{\ell} \omega}$ (right) for the identity mapping (blue) and the mapping with equidistant control points (red).

identity mapping

\begin{tabular}{ll}
\hline$\kappa(\mathbf{S})$ & $2.11 \cdot 10^{2}$ \\
bound & $1.68 \cdot 10^{7}$
\end{tabular}

geometry mapping with equal physical elements

8.19

$1.09 \cdot 10^{7}$

Table 5: Condition number and bound (without the factor $C$ ) for two parameterizations of the unit square.

The parameterization with equal physical elements leads to a better condition number and bound.

The case $D=3$. Again, an analogous observation can be drawn in the three-dimensional case. We consider different parameterizations of the unit cube by quadratic B-splines with the knot vector

$$
\left[0,0,0, \frac{1}{2}, \frac{3}{4}, \frac{7}{8}, \frac{15}{16}, \frac{31}{32}, \frac{63}{64}, \frac{127}{128}, \frac{255}{256}, 1,1,1\right]
$$

in all three parameter directions. We compare the identity mapping with a geometry mapping, where the coordinates of the control points are distributed uniformly.

We compare the condition number and its bound for both parameterizations in Table 6 . Again, $C$ depends on the same constants $p_{1}=p_{2}=p_{3}=2, K=4$ and $W=1$.

The parameterization with equidistant control points leads to a better condition number and bound. 

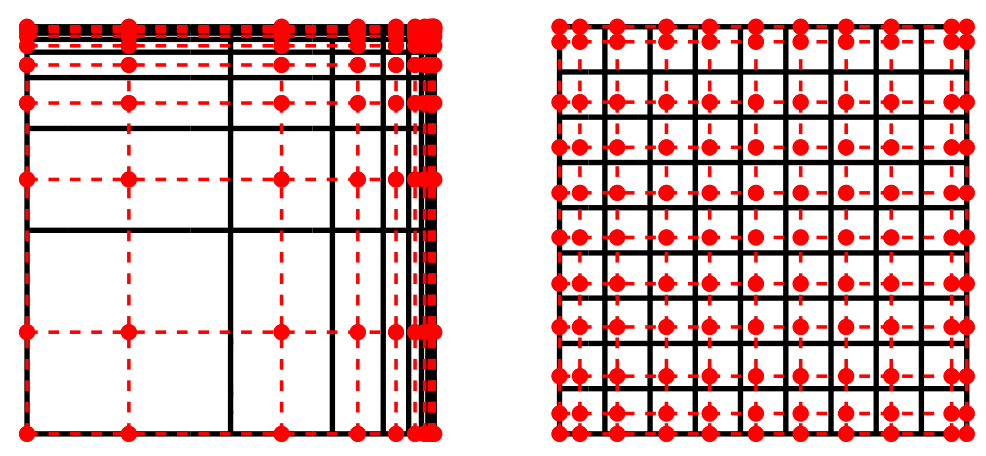

Figure 8: The parameterization of the physical domains of the unit square for the identity mapping (left) and a geometry mapping with equal elements in the physical domain (right). The red dots indicate the positions of the control points, the black lines indicate the elements in the physical domain.

\begin{tabular}{lcc} 
& identity mapping & equidistant control points \\
\hline$\kappa(\mathbf{S})$ & $1.11 \cdot 10^{3}$ & $2.79 \cdot 10^{1}$ \\
bound & $1.59 \cdot 10^{12}$ & $1.78 \cdot 10^{7}$
\end{tabular}

Table 6: Condition number and bound (without the factor $C$ ) for two parameterizations of the unit cube with non-uniform knots.

\section{Example 3 - Quantifying the parameterization quality}

We consider different parameterizations of the unit square. We use quadratic B-splines with the knot vector

$$
[0,0,0,1,1,1]
$$

in both parameter directions. Thus we need 9 control points to define the initial parameterization. We set the control points to

$$
\begin{array}{lll}
d_{0,0}=(0,0) & d_{1,0}=\left(a_{1}, 0\right) & d_{2,0}=(1,0) \\
d_{0,1}=\left(0, a_{2}\right) & d_{1,1}=\left(a_{3}, a_{4}\right) & d_{2,1}=\left(1, a_{5}\right) \\
d_{0,2}=(0,1) & d_{1,2}=\left(a_{6}, 1\right) & d_{2,2}=(1,1) .
\end{array}
$$

In order to preserve the shape of the unit square, the variables $a_{i}, i=1, \ldots, 6$ should be chosen in the open interval $(0,1)$. We generate uniformly distributed random numbers $a_{i}$ between 0 and 1 . Then we apply a uniform refinement of the geometry three times. For every parameterization constructed in this way we compute the condition number and its bound as described in the previous sections. In Fig. 9 we show the condition number and the coefficient of the computed bound for 1000 randomly constructed parameterizations. 
The points do not lie on a straight line. The more the random parameterization deviates from the identity parameterization, the higher is the deviation of data points from a straight line. However, all data points lie in a bounded region which forms roughly a cone with apex at the optimum. Morever, both boundaries are lines with positive slope. The parameterization with the smallest bound corresponds to the parameterization with the smallest condition number.

For parameterizations with higher condition numbers, we cannot directly compare two parameterizations by just considering the bound for the condition number. A parameterization with a smaller bound does not necessarily have a smaller condition number. However, both numbers are strongly related to each other.

The example demonstrates that the constructed bound reflects the quality of the parameterization in the sense that small bounds are correlated with small condition numbers. Therefore one should try to minimize the bound in order to obtain a parameterization with a small condition number.

In Fig. 9 we highlighted the data of three special parameterizations. The leftmost point indicates the identity mapping which is obviously the best parameterization in this example. The middle point indicates some random parameterization with an average condition number and bound. The rightmost highlighted point indicates a "bad" parameterization with a high condition number and bound. In Fig. 10 we show the parameterizations of the physical domain corresponding to the highlighted points in Fig. 9.

\section{Example 4 - Investigation of the effect of singularities}

We present an example which identifies the limitations of the bound: When we approach a singular situation with several coinciding control points, then the bound grows significantly faster than the condition number.

Let $D=1$ and consider a B-spline parameterization of the unit interval with the uniform knot vector

$$
\left[0,0,0, \frac{1}{9}, \frac{2}{9}, \frac{3}{9}, \frac{4}{9}, \frac{5}{9}, \frac{6}{9}, \frac{7}{9}, \frac{8}{9}, 1,1,1\right]
$$

degree $p=2$ and control points

$$
\begin{aligned}
& d_{0}=0, d_{1}=\frac{1}{18}, d_{2}=\frac{3}{18}, d_{3}=\frac{5}{18}, d_{4}=\frac{1}{2}-\tau, d_{5}=\frac{1}{2} \\
& d_{6}=\frac{1}{2}+\tau, d_{7}=\frac{13}{18}, d_{8}=\frac{15}{18}, d_{9}=\frac{17}{18}, d_{10}=1
\end{aligned}
$$




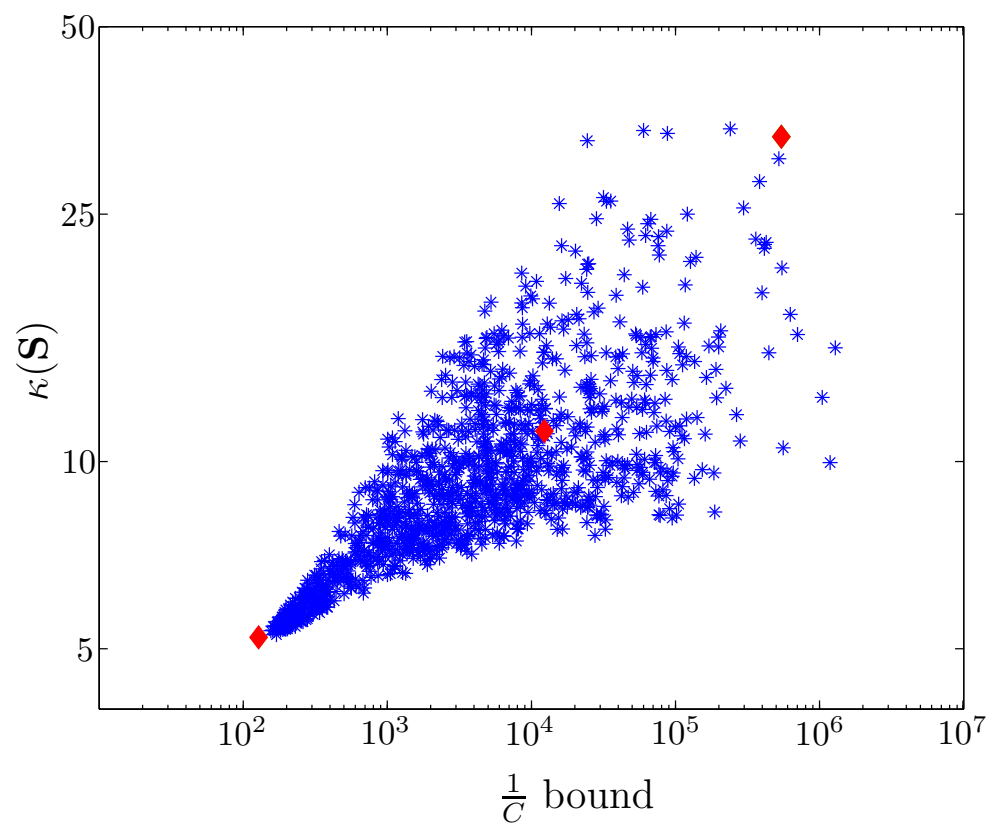

Figure 9: Doubly logarithmic plot for the condition number (vertical axis) and the coefficient of the bound (horizontal axis) for 1000 randomly constructed parameterizations of the unit square. The highlighted points correspond to the parameterizations shown in Fig. 10.
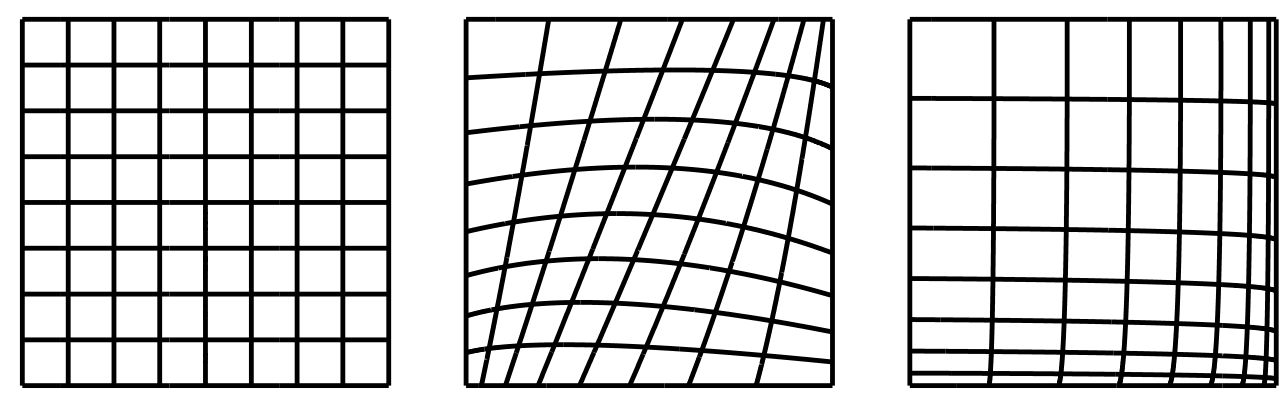

Figure 10: Physical domain of the unit square constructed with a uniform parameterization (left), an average parameterization (middle) and a "bad" parameterization with a high condition number and a high bound (right).

which depend on a parameter $\tau$. If $\tau$ tends to 0 , then the three inner control points $d_{4}, d_{5}$ and $d_{6}$ collapse into a single point. Since we have quadratic B-splines as basis functions, this leads to exactly one nearly vanishing element in the physical domain.

We vary the parameter $\tau$ and examine the behavior of the condition number and its bound. Table 7 presents different values of $\tau=\frac{1}{9} \cdot \frac{1}{2^{n}}$, the corresponding values of the 
condition number, its bound and the ratios of consecutive values. For $n=0$ we obtain $\tau=\frac{1}{9}$ and hence the identity parameterization. For the sake of brevity we display only the coefficients of the bounds in the table. Each coefficient is multiplied by a constant $C$, which depends on identical values of $K, W$ and $p$.

\begin{tabular}{c|cc|cc}
$\tau$ & $\kappa(\mathbf{S})$ & $\begin{array}{c}\text { ratio to previous } \\
\text { distortion level }\end{array}$ & bound & $\begin{array}{c}\text { ratio to previous } \\
\text { distortion level }\end{array}$ \\
\hline$\frac{1}{9}$ & 12.69 & & $8.10 \cdot 10^{1}$ & \\
$\frac{1}{9} \cdot \frac{1}{2}$ & 19.93 & 1.57 & $3.24 \cdot 10^{2}$ & 4 \\
$\frac{1}{9} \cdot \frac{1}{2^{2}}$ & 33.92 & 1.70 & $1.30 \cdot 10^{3}$ & 4 \\
$\frac{1}{9} \cdot \frac{1}{2^{3}}$ & 58.08 & 1.71 & $5.18 \cdot 10^{3}$ & 4 \\
$\frac{1}{9} \cdot \frac{1}{2^{4}}$ & 100.77 & 1.73 & $2.07 \cdot 10^{4}$ & 4 \\
$\frac{1}{9} \cdot \frac{1}{2^{5}}$ & 179.41 & 1.78 & $8.29 \cdot 10^{4}$ & 4 \\
$\frac{1}{9} \cdot \frac{1}{2^{6}}$ & 330.50 & 1.84 & $3.32 \cdot 10^{5}$ & 4 \\
$\frac{1}{9} \cdot \frac{1}{2^{7}}$ & 628.15 & 1.90 & $1.33 \cdot 10^{6}$ & 4
\end{tabular}

Table 7: Condition number, bound (without the constant C) and ratios for different levels of distortion.

When we divide $\tau$ by 2 , the condition number is multiplied approximately by 2 , while the bound is multiplied by 4 . Thus the bound grows twice as fast as the condition number. We want to identify the step of our estimates which causes this behavior.

We denote by $\mathbf{S}_{\tau}$ the global stiffness matrix for a parameterization with the variable $\tau$, by $\mathbf{S}_{\tau / 2}$ we denote the global stiffness matrix for a parameterization where we have the parameter $\tau / 2$. The same notation is used for the global mass matrix, for the element stiffness and element mass matrices and for the geometry mapping $G$.

First we consider the maximum eigenvalue of the stiffness matrix and its bound. We obtain

$$
\lambda_{\max }\left(\mathbf{S}_{\tau / 2}\right) \approx 2 \lambda_{\max }\left(\mathbf{S}_{\tau}\right), \quad \lambda_{\max }\left(\mathbf{S}_{\tau / 2}^{\ell}\right) \approx 2 \lambda_{\max }\left(\mathbf{S}_{\tau}^{\ell}\right) \quad \text { and } \quad \frac{1}{\omega_{\min , \tau / 2}^{\ell}} \approx 2 \frac{1}{\omega_{\min , \tau}^{\ell}},
$$

which confirms that this part of the bound is asymptotically correct.

However, this is no longer true for the minimum eigenvalue of the stiffness matrix. We observe that

$$
\lambda_{\min }\left(\mathbf{S}_{\tau / 2}\right) \approx \lambda_{\min }\left(\mathbf{S}_{\tau}\right), \quad \lambda_{\min }\left(\mathbf{M}_{\tau / 2}\right) \approx \lambda_{\min }\left(\mathbf{M}_{\tau}\right) \text { and } \lambda_{\min }\left(\mathbf{M}_{\tau / 2}^{\ell}\right) \approx \frac{1}{2} \lambda_{\min }\left(\mathbf{M}_{\tau}^{\ell}\right)
$$


Hence, we lose the asymptotic correctness for $\tau \rightarrow 0$ when we estimate the minimum eigenvalue of the global mass matrix by the minimum of the minimum eigenvalue of the element mass matrices. In this example, these two expressions do not scale with the same factor, which leads to the different behavior of the condition number and its bound.

\section{Example 5 - Comparing different parameterizations}

We will conclude this section by giving a three-dimensional example of a real-world object. In the framework of the EXCITING ${ }^{1}$ project, one of our tasks was to construct a volumetric parameterization of the water passage of a turbine. The parameterization of the domain provided the first step towards an isogeometric CFD simulation in order to compute the velocity and the pressure of the flowing water. Since the domain of the water passage has a rather complicated shape, it is divided into several patches that are topologically equivalent to a cube. Each patch is parameterized individually. The final parameterization consists of 8 patches, that fit together with $\mathcal{C}^{0}$-continuity. Fig. 11 shows an illustration of a Kaplan turbine and the parameterization of the water passage between two consecutive blades.
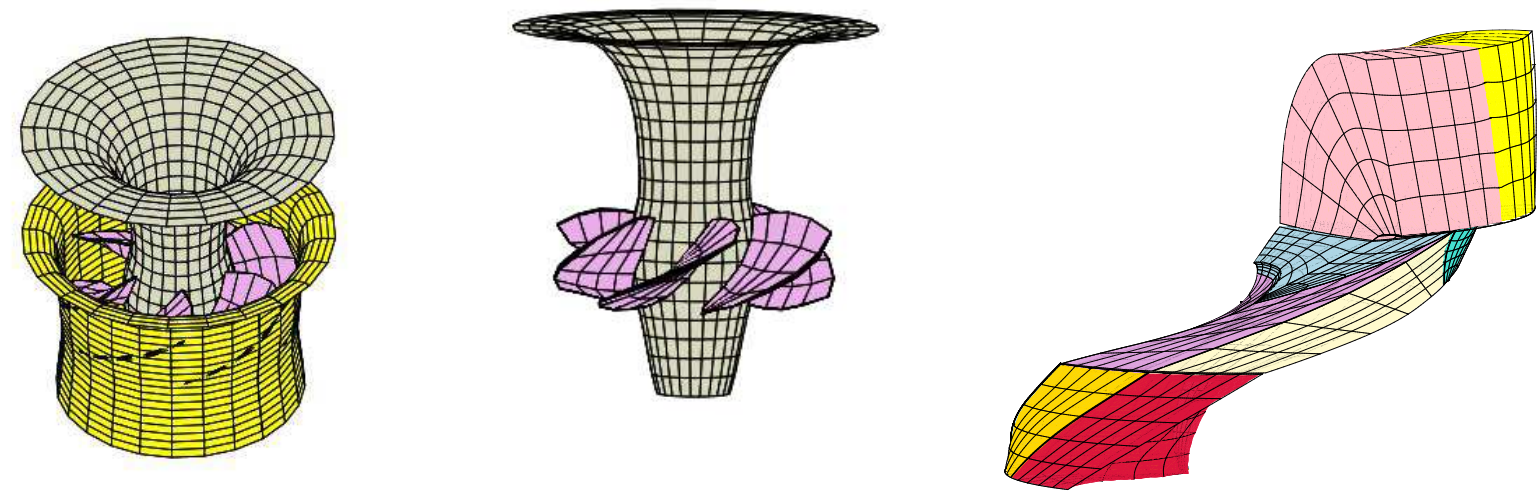

Figure 11: Illustration of a Kaplan turbine with 6 blades (left and middle). Parameterization of the water passage between two consecutive blades, consisting of 8 patches, that are pieced together with $\mathcal{C}^{0}$-continuity (right).

\footnotetext{
${ }^{1}$ Further information is available at the project web page www.exciting-project.eu
} 
Due to the complex shape of the domain we obtain several patches that have a nice shape, such as the orange patch in the bottom, and others that look kind of distorted, like the blue patch in the middle. In Fig. 12 we display these two patches. It is interesting to compare the condition number of the stiffness matrix and its bound for those two different parameterizations, in order to explore if the intuition of a "good" versus a "bad" patch is consistent with the numerical computations.
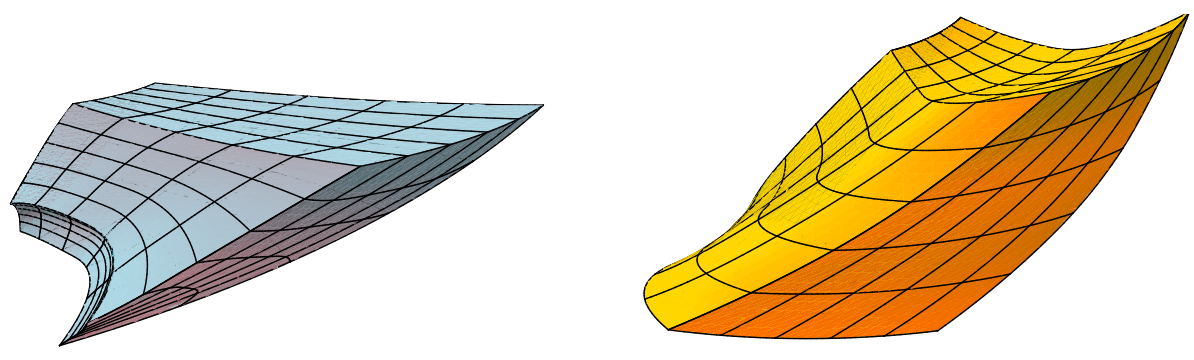

Figure 12: Two patches of the 8-patch parameterization of the water passage. The blue patch (left) is distorted in one parameter direction, while the orange patch (right) has a rather uniform shape.

Both patches are parameterized using the same degrees and knot vectors in the three parameter directions, respectively. Hence, the constants $C$ are the same for both patches. We used quadratic B-splines with $(5 \times 6 \times 4)$ control points for each patch. More details, also concerning the construction of the patches, can be found in [37]. Table 8 displays the condition numbers $\kappa(\mathbf{S})$ and the bounds for the two patches of the multi-patch-parameterization of the water passage. The patch displayed in orange leads to a much better condition number and bound than the distorted patch displayed in blue. Hence - in this situation - the intuition is in accordance with the numerical results.

\begin{tabular}{lcc} 
& blue patch & orange patch \\
\hline$\kappa(\mathbf{S})$ & 32.15 & 24.86 \\
bound (divided by $C)$ & $1.34 \cdot 10^{5}$ & $4.39 \cdot 10^{3}$
\end{tabular}

Table 8: Condition number and bound (without the factor $C$ ) for two different patches of a multi-patch parameterization.

It is also obvious that the bound exaggerates the effect of the worse parameterization of the blue patch, which may be due to facts described in the previous example. 


\section{Conclusions}

In this paper we have investigated the condition number of the stiffness matrix for the Poisson equation. More precisely, we derived bounds for the condition number for dimensions $D \leq 3$. The given bounds are invariant under scaling and asymptotically optimal with respect to uniform refinement. In general, the bounds depend on the lengths of the knot spans in the parameter domain and some term depending on the geometry mapping. A parameterization can be optimized, such that non-uniform knot vectors can be compensated by a suitable geometry mapping.

In general we can state that the size of the elements in the physical domain should not vary too much, such that we do not get very small elements. Furthermore, the parameter lines should be as orthogonal as possible and the parameter directions (in the two-dimensional case and in the three-dimensional case) should approximately have the same length. Computing the bound for the condition number can help to decide where a parameterization should be improved.

The given bounds can be applied to regular parameterizations with $\omega=|\operatorname{det} \mathbf{J}| \neq 0$ for all parameter values. As a limitation of the bound, it exaggerates the effect of near singular parameterizations. This, however, should be no problem when using it in practice, since it may help to avoid singular and near-singular parameterizations. Future work may be devoted to possible improvements of this bound, to more precise estimates (instead of bounds) of the condition number, to the evaluation of the constant $C$, and to the applications of this bound for optimizing domain parameterizations in IGA.

\section{Acknowledgments}

This research was supported by the European Commission through the project "Exact Geometry Simulation for Optimized Design of Vehicles and Vessels" (EXCITING, GA no. 218536) and by the Austrian Science Fund through the NFN "Geometry + Simulation" (S117). The authors thank the reviewers for their valuable comments which have helped to improve this paper.

\section{References}

[1] T. J. R. Hughes, J. A. Cottrell, Y. Bazilevs, Isogeometric analysis: CAD, finite elements, NURBS, exact geometry and mesh refinement, Computer Methods in Applied Mechanics and Engineering 194 (2005) 4135 - 4195. 
[2] Y. Bazilevs, L. Beirão da Veiga, J. A. Cottrell, T. J. R. Hughes, G. Sangalli, Isogeometric analysis: Approximation, stability and error estimates for h-refined meshes, Mathematical Models and Methods in Applied Sciences 16 (2006) 1031 - 1090.

[3] L. Beirão da Veiga, A. Buffa, J. Rivas, G. Sangalli, Some estimates for h-p-krefinement in Isogeometric Analysis, Numerische Mathematik 118 (2011) 271-305.

[4] L. Beirão da Veiga, D. Cho, G. Sangalli, Anisotropic NURBS approximation in isogeometric analysis, Computer Methods in Applied Mechanics and Engineering 209-212 (2012) 1-11.

[5] T. J. R. Hughes, A. Reali, G. Sangalli, Efficient quadrature for NURBS-based isogeometric analysis, Computer Methods in Applied Mechanics and Engineering 199 (2010) $301-313$.

[6] W. G. Strang, G. Fix, An Analysis of the Finite Element Method, Prentice-Hall, New Jersey, 1973.

[7] O. C. Zienkiewicz, R. L. Taylor, J. Z. Zhu, The Finite Element Method: Its Basis and Fundamentals, Elsevier Science, Oxford, 2005.

[8] S. C. Brenner, R. L. Scott, The Mathematical Theory of Finite Element Methods, Springer, 2005.

[9] R. E. Bank, L. R. Scott, On the conditioning of finite element equations with highly refined meshes, SIAM J. Numer. Anal. 26 (1989) 1383-1394.

[10] N. Hu, X.-Z. Guo, I. N. Katz, Bounds for eigenvalues and condition numbers in the p-version of the finite element method, Mathematics of Computation 67 (1998) $1423-1450$.

[11] Q. Du, D. Wang, L. Zhu, On mesh geometry and stiffness matrix conditioning for general finite element spaces, SIAM J. Numer. Anal. 47 (2009) 1421-1444.

[12] M. Aigner, C. Heinrich, B. Jüttler, E. Pilgerstorfer, B. Simeon, A.-V. Vuong, Swept Volume Parameterization for Isogeometric Analysis, in: E. Hancock, R. Martin (Eds.), The Mathematics of Surfaces XIII, volume 5654 of Lecture Notes in Computer Science, Springer, 2009, pp. 19-44. 
[13] E. Cohen, T. Martin, R. M. Kirby, T. Lyche, R. F. Riesenfeld, Analysis-aware modeling: Understanding quality considerations in modeling for isogeometric analysis, Computer Methods in Applied Mechanics and Engineering 199 (2010) 334 - 356.

[14] T. Martin, E. Cohen, R. M. Kirby, Volumetric parameterization and trivariate Bspline fitting using harmonic functions, Computer Aided Geometric Design 26 (2009) $648-664$.

[15] G. Xu, B. Mourrain, R. Duvigneau, A. Galligo, Optimal analysis-aware parameterization of computational domain in isogeometric analysis, Proc. of Geometric Modeling and Processing (2010) 236-254.

[16] G. Xu, B. Mourrain, R. Duvigneau, A. Galligo, Parameterization of computational domain in isogeometric analysis: Methods and comparison, Computer Methods in Applied Mechanics and Engineering 200 (2011) 2021-2031.

[17] G. Xu, B. Mourrain, R. Duvigneau, A. Galligo, Analysis-suitable volume parameterization of multi-block computational domain in isogeometric applications, ComputerAided Design 45 (2013) 395-404.

[18] T. Takacs, B. Jüttler, Existence of Stiffness Matrix Integrals for Singularly Parameterized Domains in Isogeometric Analysis, Computer Methods in Applied Mechanics and Engineering 200 (2011) 3568 - 3582.

[19] T. Takacs, B. Jüttler, $H^{2}$ regularity properties of singular parameterizations in isogeometric analysis, Graphical Models 74 (2012) 361-372.

[20] A. Evgrafov, A. R. Gersborg, J. Gravesen, D. M. Nguyen, P. N. Nielsen, Isogeometric analysis and shape optimisation, Proceedings of 23rd Nordic Seminar on Computational Mechanics (2010) 14-17.

[21] D. M. Nguyen, A. Evgrafov, A. R. Gersborg, J. Gravesen, Isogeometric shape optimization of vibrating membranes, Computer Methods in Applied Mechanics and Engineering 200 (2011) 1343-1353.

[22] D. M. Nguyen, A. Evgrafov, J. Gravesen, Isogeometric shape optimization for electromagnetic scattering problems, Progress in Electromagnetic Research (PIER) B 45 (2012) 117-146. 
[23] X. Qian, Full Analytical Sensitivities in NURBS based Isogeometric Shape Optimization, Computer Methods in Applied Mechanics and Engineering 199 (2010) 2059 2071.

[24] W. A. Wall, M. A. Frenzel, C. Cyron, Isogeometric structural shape optimization, Computer Methods in Applied Mechanics and Engineering 197 (2008) 2976 - 2988.

[25] Y.-D. Seo, H.-J. Kim, S.-K. Youn, Shape optimization and its extension to topological design based on isogeometric analysis, International Journal of Solids and Structures 47 (2010) $1618-1640$.

[26] A. Pospíšilová, M. Lepš, D. Rypl, B. Patzák, Shape Optimization using Isogeometric Analysis and Particle Swarm Optimization, In B.H.V. Topping, (Editor), Proceedings of the Eleventh International Conference on Computational Structures Technology, Civil-Comp Press, Stirlingshire (UK) Paper 220 (2012).

[27] S. Lipton, J. A. Evans, Y. Bazilevs, T. Elguedj, T. J. R. Hughes, Robustness of isogeometric structural discretizations under severe mesh distortion, Computer Methods in Applied Mechanics and Engineering 199 (2010) 357 - 373.

[28] K. Gahalaut, S. Tomar, Condition number estimates for matrices arising in the isogeometric discretizations, RICAM report 2012-23 (2012) 1-38.

[29] C. de Boor, A Practical Guide to Splines, Springer Verlag, New York, 1978.

[30] H. Prautzsch, W. Boehm, M. Paluszny, Bézier and B-Spline Techniques, Springer, New York, 2002.

[31] J. Hoschek, D. Lasser, Fundamentals of Computer Aided Geometric Design, A. K. Peters, Ltd., Natick, 1993.

[32] L. Piegl, W. Tiller, The NURBS book, Springer, London, 1995.

[33] J. A. Cottrell, T. J. R. Hughes, Y. Bazilevs, Isogeometric Analysis. Towards Integration of CAD and FEA, Wiley \& Sons, Ltd., Chichester, 2009.

[34] Y. Ikebe, T. Inagaki, S. Miyamoto, The Monotonicity Theorem. Cauchy's Interlace Theorem, and the Courant-Fischer Theorem, The American Mathematical Monthly 94 (1987) 352-354. 
[35] J. Nedelec, Acoustic and Electromagnetic Equations, Integral Representations for Harmonic Problems, Springer, New York, 2001.

[36] K. Gahalaut, J. Kraus, S. Tomar, Multigrid methods for isogeometric discretization, Comput. Methods Appl. Mech. Engrg. 253 (2012) 413-425.

[37] E. Pilgerstorfer, Construction and Analysis of Volume Parameterizations for Isogeometric Analysis, Ph.D. thesis, Johannes Kepler University Linz, Austria, 2013. 